\title{
Computational Design of Nanostructural Color for Additive Manufacturing
}

\author{
THOMAS AUZINGER, Institute of Science and Technology Austria, Austria \\ WOLFGANG HEIDRICH, King Abdullah University of Science and Technology, Kingdom of Saudi Arabia \\ BERND BICKEL, Institute of Science and Technology Austria, Austria
}
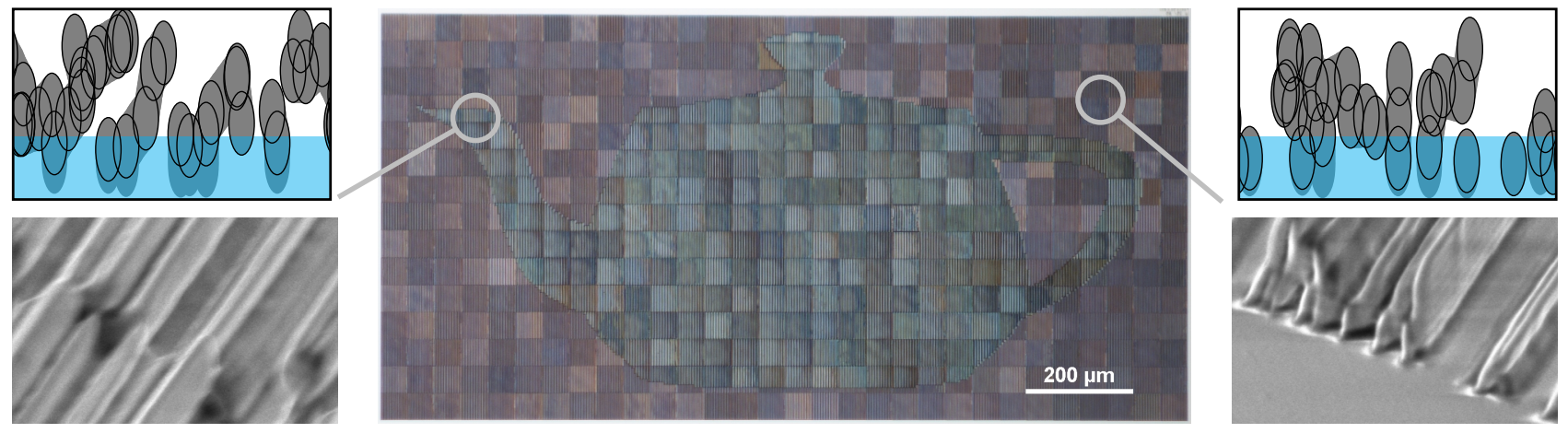

Fig. 1. (Center) Microscope image of a structurally colorized surface with the corresponding nanostructures of the fore- and background depicted left and right of it. (Top) The cross-sectional profiles of both transparent structures were designed with our method using full electromagnetic simulation. (Bottom) Micrographs of the fabricated nanostructures with a length scale of $5 \mu \mathrm{m}$ across the image diagonal.

\begin{abstract}
Additive manufacturing has recently seen drastic improvements in resolution, making it now possible to fabricate features at scales of hundreds or even dozens of nanometers, which previously required very expensive lithographic methods. As a result, additive manufacturing now seems poised for optical applications, including those relevant to computer graphics, such as material design, as well as display and imaging applications.

In this work, we explore the use of additive manufacturing for generating structural colors, where the structures are designed using a fabrication-aware optimization process. This requires a combination of full-wave simulation, a feasible parameterization of the design space, and a tailored optimization procedure. Many of these components should be re-usable for the design of other optical structures at this scale.

We show initial results of material samples fabricated based on our designs. While these suffer from the prototype character of state-of-the-art fabrication hardware, we believe they clearly demonstrate the potential of additive nanofabrication for structural colors and other graphics applications.
\end{abstract}

Authors' addresses: Thomas Auzinger, Institute of Science and Technology Austria, Am Campus 1, 3400, Klosterneuburg, Austria, thomas.auzinger@ist.ac.at; Wolfgang Heidrich, King Abdullah University of Science and Technology, Al Khwarizmi Building 1, 23955-6900, Thuwal, Kingdom of Saudi Arabia, wolfgang.heidrich@kaust.edu.sa; Bernd Bickel, Institute of Science and Technology Austria, Am Campus 1, 3400, Klosterneuburg, Austria, bernd.bickel@ist.ac.at.

Permission to make digital or hard copies of all or part of this work for personal or classroom use is granted without fee provided that copies are not made or distributed for profit or commercial advantage and that copies bear this notice and the full citation on the first page. Copyrights for components of this work owned by others than the author(s) must be honored. Abstracting with credit is permitted. To copy otherwise, or republish, to post on servers or to redistribute to lists, requires prior specific permission and/or a fee. Request permissions from permissions@acm.org.

(C) 2018 Copyright held by the owner/author(s). Publication rights licensed to Association for Computing Machinery.

0730-0301/2018/8-ART159 \$15.00

https://doi.org/10.1145/3197517.3201376
CCS Concepts: • Applied computing $\rightarrow$ Computer-aided manufacturing; • Hardware $\rightarrow$ Emerging optical and photonic technologies;

Additional Key Words and Phrases: structural colorization, appearance, multiphoton lithography, direct laser writing, computational fabrication, computational design, shape optimization, FDTD, diffraction, Nanoscribe

\section{ACM Reference Format:}

Thomas Auzinger, Wolfgang Heidrich, and Bernd Bickel. 2018. Computational Design of Nanostructural Color for Additive Manufacturing. ACM Trans. Graph. 37, 4, Article 159 (August 2018), 16 pages. https://doi.org/10.1145/ 3197517.3201376

\section{INTRODUCTION}

In contrast to pigment-based colors - where spectral components of incident light are absorbed by the pigment material - structural colorization arises from the interaction of light with micro- and nanostructures. Variations of the refractive index at the length scale of the light's wavelength cause interference effects that lead to fascinating optical effects such as iridescence. Designing such colorization is challenging, however, since the wave nature of light has to be taken into account when simulating its interplay with nanostructures. Furthermore, realizing such structures requires elaborate methods from nanofabrication. Owning to these complications, linking computational methods and advanced manufacturing is essential for exploring structural colorization, but so far has received little attention from the scientific community, compared to recent progress in designing structures for elastic, auxetic, translucent, or reflective materials [Bermano et al. 2017; Hullin et al. 2013].

In recent years, an advanced additive manufacturing technology has reached commercial maturity, which permits the fabrication of sub-micrometer structures. Multiphoton lithography - also known 


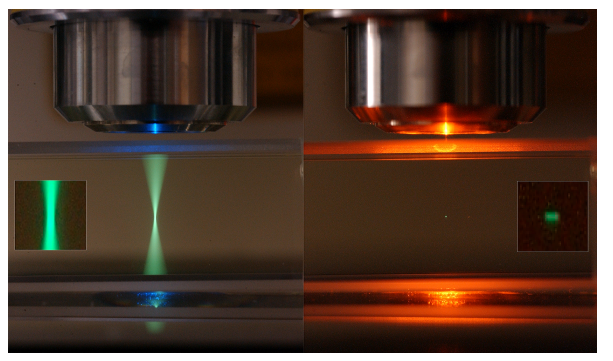

Fig. 2. Comparison between one- and two-photon fluorescence. Only at the very center of the two-photon focus region (right), the light intensity is sufficiently high to initiate material polymerization, which enables the fabrication of free-form sub-micrometer structures. Image by Steve Ruzin and Holly Aaron, UC Berkeley.

as direct laser writing (DLW) - acts similar to stereolithography (SLA) by using light to solidify a liquid photo-polymer. However, in contrast to SLA, it moves a small focus region along arbitrary three-dimensional paths through the photoresist as shown in Figure 2 (right). Only inside the focus spot, light intensity is high enough to enable the absorption of two photons in rapid succession whose combined energy surpass the polymerization threshold for material solidification. Although more complex than traditional 3D printing, this method still retains many of its convenient aspects, above all its suitability for fabricating shapes with complex geometries. The cost of a DLW setup is in the same range as modern multi-material 3D printers and at least an order of magnitude lower than for conventional lithography devices in nanofabrication. Potential applications of this technology lie in cell biology, microfluidics, general micromachinery, and - of special interest to us - optics.

In this paper, we present a computational design tool for the creation of transparent nanostructures with feature sizes in the range of $100 \mathrm{~s}$ of $\mathrm{nm}$ that realize simple colorization of light transmitted through them. The system is built around a full electromagnetic simulation of light in order to faithfully capture wave- and polarization-dependent effects that approximations to light transport cannot handle. We take fabrication constraints of multiphoton lithography into account to ensure the feasibility of fabricating the designs. In summary, we present the following contributions:

- A formulation of the aforementioned design system as a nonlinear optimization framework exploiting the Helmholtz reciprocity (Section 4).

- A parameterization of nanostructures tailored to the fabrication constraints of DLW (Section 4.2).

- An adaptation of the electromagnetic finite-difference timedomain (FDTD) simulation method to our setting, which includes gradient computations via the adjoint state method and a novel memory-efficient signal reconstruction method (Section 5).

- A graph-based method for structural reinforcement to ensure the mechanical stability of the design (Section 6.1).

After a description of our fabrication process using DLW (Section 6), we demonstrate the capabilities of our design system in a number of optimization results as well as fabricated samples (Section 7).

\section{RELATED WORK}

\subsection{Appearance Design for Fabrication}

There has been a considerable amount of work for computationally designing and reproducing objects and materials with desired visual properties in computer graphics and beyond [Hullin et al. 2013]. Approaches for reproducing bidirectional reflectance distribution functions (BRDFs) based on a spatial combination of inks [Matusik et al. 2009], surface structures significantly larger than the wavelength of light [Lan et al. 2013; Weyrich et al. 2009], or custom orientation of magnetic flakes in ultraviolet (UV)-curable resin [Pereira et al. 2017] have been proposed. Simulating a grid of binary steps with height difference $\approx 100 \mathrm{~nm}$ by using wave optics, Levin et al. [2013] fabricated surface patterns with a monochrome reflectance behavior dependent on the incident light direction. For reproducing color and texture with $3 \mathrm{D}$ printers, methods for spatial dithering [Brunton et al. 2015] and optimization of discrete voxel distributions [Babaei et al. 2017; Elek et al. 2017] have been investigated. Inverse problems also have been targeted for fabricating desired subsurface scattering effects from a fixed set of translucent materials [Dong et al. 2010; Hašan et al. 2010] or mixtures of inks [Papas et al. 2013].

\subsection{Structural Color}

Comparison. In comparison to pigment-based colors, structural colors have various benefits: they enable more vivid colorizations and - as long as their structure is intact - they do not fade over time. Furthermore, they do not need a wide range of potentially toxic pigments but can be fabricated out of a single inert substance [Aguirre et al. 2010]. Of special interest for computer graphics is also their ability to enable exotic colorization effect, such as iridescence, or strain-dependent color variations [Burgess et al. 2013]. This comes at the cost of increased design and fabrication complexity: while conventional colors can be achieved through comparably simple and intuitive pigment mixtures, wave-optical simulations and nanofabrication are required to create structural colorizations. In this work, we want to argue, however, that these complications are mitigated by recent technological advances creating new avenues for computer graphics research.

Natural Occurrence. Many brilliant colors in nature do not arise due to light-absorbing pigmentations but due to interference effects caused by nanostructures [Kinoshita et al. 2008]. A wide variety of structures, which serve basic functions such as camouflage [Wickler 1965] or courtship [Schultz and Fincke 2009], can be observed both in flora and fauna, such as layered structures, 3D photonic crystals, and surface structures [Vukusic and Sambles 2003].

Rendering. The appearance of some common objects, for example made out of metal or plastic, is influenced by diffraction of light reflecting off microscopic surface details. Being fundamentally a wave-optical phenomenon, efficient approaches have been developed for obtaining wave-optical shading models for rendering. These methods usually assume a particular substructure, such as scratches [Werner et al. 2017], nanofacets [Holzschuch and Pacanowski 2017], or thin films [Belcour and Barla 2017]. To simulate general structures, such as ours, tools from computational electromagnetics 
are needed, which can be categorized into integral solvers, such as the boundary-element-based method of moments (MoM) [Gibson 2014], and differential solvers, such as the finite element method (FEM) [Jin 2014] and the finite-difference time-domain (FDTD) method [Taflove and Hagness 2005]. We employ the latter owing to its versatility and suitability for our setting.

Fabrication. Due to the potentially far-reaching applications of structural colorization in fields such as sensing [Pris et al. 2012], security [Lee et al. 2013], apparel [Sato et al. 2009], and pigmentation replacement [Aguirre et al. 2010], its analysis and replication has received considerable attention from the research community. Generally, the physical effect that is responsible for the colorization requires an adequate fabrication method. Deposition methods are used for thin dielectric and metallic films for etalons [Park et al. 2016], Fabry-Perot cavities [Yang et al. 2016], or thin-film stacks [Song et al. 2017]. To obtain spatially-varying colorizations or non-uniform structures, additional dry or wet etching is usually required. The former allows the realization of top-coated pillars for 100,000 DPI color images [Kumar et al. 2012] while the latter was used to create sponge-like metallic structures for wear-resistant structural colorization [Galinski et al. 2016]. We refer to recent surveys for a comprehensive overview [England and Aizenberg 2017; Fu et al. 2016]. Since we emphasize ease of fabrication in this work, we forgo such elaborate (often vacuum-dependent) fabrication methods and utilize direct laser writing (DLW). While this poses restrictions in terms of resolution and materials, it offers full freeform flexibility and is not restricted to heightfields [Johansen et al. 2015].

Design. The common approach for creating suitable structures for structural colorizations is either to replicate designs from nature [Zyla et al. 2018] or to emphasize a certain physical effect [Kumar et al. 2012]. In both cases, the structures usually have only a few degrees of freedom and the corresponding (often continuous) parameter space can be efficiently searched either by simulation [Vargo 2017] or fabrication [Kumar et al. 2012]. An alternative approach is to parameterize very general structures with many degrees of freedom and to employ some computational optimization to find suitable parameters that realize a desired colorization. The most general approach of topology optimization allows the treatment of complex physical effects, e.g., band-gap design of photonic crystals [Yi and Youn 2016] or - similar to our work - structural color [Andkjær et al. 2013], but generally yield designs in the form of complex patterns that are not easily amendable to fabrication. An introduction to topology optimization for fabrication and a broad overview of applications can be found in [Wu et al. 2017]. We employ an intermediate approach, where we use a structure parameterization that constrains the design space to a fabricable subspace. For structural colorization, such a restriction was used for height field design [Johansen et al. 2014] and arrangements of rings that stay transparent under omnidirectional illumination [Hsu et al. 2015]. However, both works do not take realistic fabrication constraints into account.

\subsection{Multiphoton Lithography}

Although discovered in 1965 [Pao and Rentzepis 1965], it took many decades of development for DLW to become practical. The ability to generate free-form nanostructures has a wide field of application, and in recent years, it has been applied to tissue engineering [Raimondi et al. 2012], micromachinery and microrobotics [MedinaSánchez et al. 2015; Villangca et al. 2016], as well as the creation of superstrong [Gu and Greer 2015] and resilient materials [Meza et al. 2015]. In the field of structural color, simple photonic crystals in the form of woodpile structures were created by Mizeikis [2014] while Nawrot et al. [2013] created diffraction gratings from regular pillar arrangements. Both approaches exhibit a limited design space with two continuous degrees of freedom, which allow the creation of a color palette. For a lesser degree, this also holds for the work of Zyla et al. [2018], who replicate the thin-layer stacks of the Morpho butterfly using DLW. To our knowledge, our work is the first that addresses the inverse design of general freeform DLW-fabricable nanostructures for structural colorization.

\section{DESIGN CONSIDERATIONS}

Our goal is to design transparent structures with features in the 100 s of nanometer range that realize a desired colorization of transmitted light. Given a well-specified incident illumination, specific colors should be observable when viewing these structures from a macroscopic distance. However, it is not possible to realize arbitrary spectral angular color distribution even with the most advanced fabrication methods due to inherent physical constraints. In the following, we outline the main consequences of our choices regarding fabrication and simulation methods.

Fabrication Considerations. The ease of use that comes with direct laser writing (DLW) entails restrictions in terms of $i$ ) the achievable resolution and $\mathrm{ii}$ ) the available materials. While feature generation down to $50 \mathrm{~nm}$ has been reported for experimental multiphoton lithography setups [Emons et al. 2012], industrial solutions - such as the one we are using - have minimal feature sizes several times this size. Since structural colorization requires (sub-)wavelengthsized structures, we need to operate the fabrication device at its resolution limit. Thus, we parameterize our structures in a way that directly corresponds to paths along which the liquid photoresist is polymerized. In contrast, conventional additive manufacturing is generally used to fabricate objects much larger than the paths or layers of each process step.

Compatible photoresists for DLW are transparent non-metallic materials with a refractive index of around 1.5. Together with the refractive index of air at around 1.0, this generates a low contrast structure when compared with other commonly used materials for optical purposes, such as $\mathrm{TiO}_{2}$ with a refractive index of around 2.6. As a consequence, we face a reduced design space when compared with far more complex fabrication methods, such as the metal-coated posts with 50-150 nm diameter for the full color 100,000 DPI result of Kumar et al. [2012]. However, we still obtain interesting colorization in this challenging setting.

Simulation Considerations. Due to the freeform shape of our structures and their length scale on the same order as visible light, we employ full electromagnetic FDTD simulations to determine their influence on transmitted light (see Section 5.2 for details). The high computational cost of such simulations requires us to reduce both 
the number of simulations as well as the per-simulation runtime requirements to ensure a practical design system. Their number is reduced by simulating all wavelengths, polarizations and incident light directions simultaneously; only the outgoing light directions have to be simulated independently (though they can run in parallel). Additionally, we apply two simplifications to our setting in order to reduce the size of the region in which such a simulation is required. On the one hand, we limit the control over the colorization to one incident plane by assuming our structure consists of a $2 \mathrm{D}$ profile that is extruded along the surface. This reduces the polynomial complexity of the FDTD from fourth to third order in the grid resolution. On the other hand, we assume that our structure is periodically tiled across the surface and we deduce its large-scale behavior by simulating a single period.

\section{INVERSE STRUCTURE DESIGN}

For a given colorization objective, our goal is to design a nanostructure that realizes this effect when light passes through it. In the following sections, we describe the formulation of the objective as well as our chosen parameterization of the structure. Subsequently, we define the optimization problem and its constraints.

\subsection{Colorization Objective}

The optical properties of a 2D nanostructure can be described by its spectral bidirectional scattering distribution function (BSDF) $f_{\mathrm{s}}\left(\theta_{\mathrm{i}}, \theta, \lambda\right)$, which relates the amount of light with wavelength $\lambda$ incoming from direction $\theta_{\mathrm{i}}$ with the light of the same wavelength outgoing to direction $\theta$. Since we are interested in the colorization of transmissive light, we focus on the transmittance part of the BSDF, which is given by the spectral bidirectional transmittance distribution function (BTDF) $f_{\mathrm{t}}\left(\theta_{\mathrm{i}}, \theta, \lambda\right)$. For a viewing direction $\theta$, the observable light in terms of spectral radiance $L(\theta, \lambda)$ is given as

$$
L(\theta, \lambda)=\int_{\mathcal{H}} f_{\mathrm{t}}\left(\theta_{\mathrm{i}}, \theta, \lambda\right) L_{\mathrm{i}}\left(\theta_{\mathrm{i}}, \lambda\right) \cos \left(\theta_{\mathrm{i}}\right) d \theta_{\mathrm{i}},
$$

where radiance $L_{\mathrm{i}}$ of all incoming light directions $\theta_{\mathrm{i}}$ is accumulated.

The observable color $\mathbf{c}(\theta)$ can be directly obtained via a color conversion of the associated radiant power spectrum $\phi(\theta, \lambda)$ with the help of standard color matching functions $\mathrm{m}$, i.e.,

$$
\mathbf{c}(\theta)=\int \mathbf{m}(\lambda) \phi(\theta, \lambda) d \lambda .
$$

The power spectrum constitutes an accumulation of spectral radiances over the area and the opening angle of a (virtual) sensor - both of which we assume to be small enough that the radiance can be assumed constant.

A straightforward way to define a design objective is to specify a target BTDF $\breve{f}_{\mathrm{t}}\left(\breve{\theta}_{\mathrm{i}}, \breve{\theta}, \breve{\lambda}\right)$ for appropriate samplings $\breve{\theta}_{\mathrm{i}}, \breve{\theta}$, and $\breve{\lambda}$ of its parameters. However, there are two issues with this immediate approach: $i$ ) many of the possible target specifications are unphysical and not attainable; and ii) the effects of nanostructures on different wavelengths is correlated in unintuitive ways. Thus, we specify colorization objectives as observable color distributions $\breve{\mathbf{c}}_{j}\left(\breve{\theta}_{k}\right)$ for a set of sampled view directions $\breve{\theta}_{k}$. Each objective corresponds to certain incident illumination settings as given by a spectral radiance distribution $L_{\mathrm{i}, j}\left(\theta_{\mathrm{i}}, \lambda\right)$. An example would be a cone of daylight illumination with opening angle $\alpha$, i.e.,

$$
L_{\mathrm{i}, j}\left(\theta_{\mathrm{i}}, \lambda\right)=\mathrm{D}_{65}(\lambda) \chi\left(\left|\theta_{\mathrm{i}}\right| \leq \frac{\alpha}{2}\right),
$$

with $\chi$ denoting the characteristic function and where the spectral composition is given by the $\mathrm{D}_{65}$ CIE standard illuminant.

We define the objective function $\varphi$ as

$$
\varphi(\mathbf{q})=\frac{1}{2} \sum_{L_{\mathrm{i}, j}} \sum_{\breve{\theta}_{k}}\left\|\breve{\mathbf{c}}\left(\breve{\theta}_{k}\right)-\mathbf{c}\left(\breve{\theta}_{k}, L_{\mathrm{i}, j}, \mathbf{q}\right)\right\|^{2},
$$

where we penalize deviation from the target colors $\breve{c}$ over all chosen outgoing directions $\breve{\theta}_{k}$ for all incident illuminations $L_{\mathrm{i}, j}$. The dependency on the nanostructure geometry is explicitly stated using a structure parameterization q. Details on utilized color spaces and color differences are given in the supplemental material.

Later in Section 5.1.1, we will see that due to the periodic tiling of the nanostructure, the BTDF has a very particular structure. Only a finite number of incoming and outgoing direction pairs has nonzero transmittance and the integration of Equation (1) is actually a sum over discrete incoming directions $\theta_{\mathbf{i}}(\theta, \lambda)$, which vary for each outgoing direction $\theta$ and each wavelength $\lambda$. Thus, the BTDF is actually a sum of directional delta distributions, whose formal definition introduces considerable notational overhead. We avoid this by simply using spectral transmittances $\tau\left(\theta_{\mathrm{i}}, \theta, \lambda, \mathbf{q}\right)$ to describe the relationship between in- and outgoing radiance, i.e.,

$$
L(\theta, \lambda, \mathbf{q})=\tau\left(\theta_{\mathrm{i}}, \theta, \lambda, \mathbf{q}\right) L_{\mathrm{i}}\left(\theta_{\mathrm{i}}, \lambda\right)
$$

Due to the Helmholtz reciprocity of optics, which constitutes a special case of the general reciprocity in electromagnetism, the transmittance is symmetric in the in- and outgoing angle, i.e., $\tau\left(\theta_{\mathrm{i}}, \theta, \lambda, \mathbf{q}\right)=$ $\tau\left(\theta, \theta_{\mathrm{i}}, \lambda, \mathbf{q}\right)$. This property will be essential for our simulation setting, as we will conduct them in the reciprocal setting where we inject light from the outgoing directions to compute the transmittances. Consequently, we perform a separate simulation for each outgoing direction $\breve{\theta}_{k}$, each of which computes the spectral transmittances $\tau\left(\theta_{\mathbf{i}, m}\left(\breve{\theta}_{k}, \lambda\right), \breve{\theta}_{k}, \lambda, \mathbf{q}\right)$ between $\breve{\theta}_{k}$ and all its corresponding incident directions $\theta_{\mathrm{i}, m}\left(\breve{\theta}_{k}, \lambda\right)$. Integrating the transmittances into the objective function definition yields

$$
\begin{aligned}
& \varphi(\mathbf{q})=\frac{1}{2} \sum_{L_{\mathrm{i}, j}} \sum_{\breve{\theta}_{k}} \| \breve{\mathbf{c}}\left(\breve{\theta}_{k}\right)-\int \mathbf{m}(\lambda) \\
& \sum_{m} \tau\left(\theta_{\mathrm{i}, m}\left(\breve{\theta}_{k}, \lambda\right), \breve{\theta}_{k}, \lambda, \mathbf{q}\right) L_{\mathrm{i}, j}\left(\theta_{\mathrm{i}, m}\left(\breve{\theta}_{k}, \lambda\right), \lambda\right) d \lambda \|^{2},
\end{aligned}
$$

where $m$ indexes the transmitted incident directions $\theta_{\mathrm{i}, m}$.

There is much to be said about the nature of the discrete incident directions, which can be explained by diffraction grating theory. In the same sense, the aforementioned transmittances are actually the diffraction efficiencies of these directions. For the sake of clarity as well as brevity, we omit these details here and we point the interested reader to the supplemental material.

\subsection{Structure Parameterization}

The main approaches for designing optimal material distributions can be divided into two major categories depending on how they represent the material: $i$ ) as a spatial distribution where each location is assigned local material properties, i.e., topology optimization; 


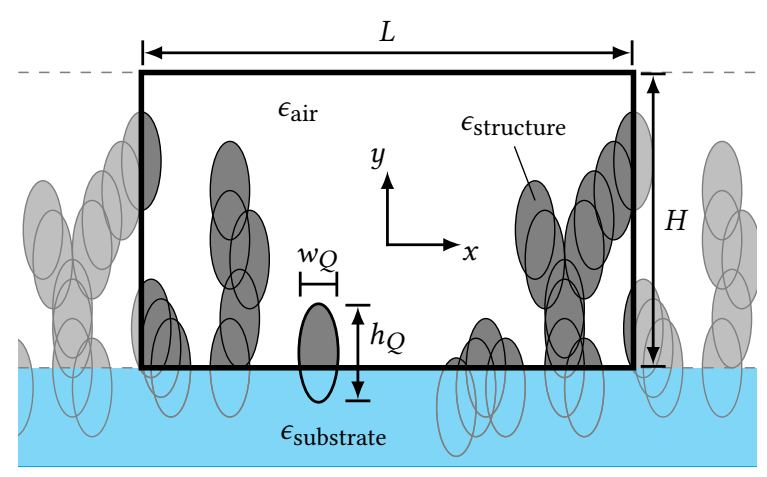

Fig. 3. Unit cell setup. A laterally tiled unit cell of length $L$ and height $H-$ located above the substrate illustrated in blue - contains an arrangement of fundamental elements, which constitute the optically active nanostructure, shown in gray. Each element represents the smallest region of material deposition - referred to as focus - with the shape of an ellipsoid of lateral diameter $w_{Q}$ and height $h_{Q}$. The permittivities $\epsilon$ of air, substrate, and structure are assigned to these regions and the background. For fabrication, this $2 \mathrm{D}$ profile is extruded along the out-of-plane $z$-direction.

and ii) as the boundary between regions of different materials, i.e., shape optimization. While the former effortlessly handles arbitrary structures only limited by the resolution of the spatial discretization, it generally assumes a continuous range of material properties. Our two-material setting violates this assumption and some form of binarization methodology would be required [Wu et al. 2017]. Furthermore, it is cumbersome to adapt our fabrication limitations (e.g., minimal feature size) to this setting. Thus, we employ shape optimization by considering the boundary between the structure material and the surrounding air and the glass substrate, all of which have uniform properties over their respective regions. Using general boundary descriptions such as level sets has the same drawback regarding fabrication limitations, however, and we choose to directly enforce these limitations by our shape representation.

We define our nanostructures as spatial arrangements of atomic elements. Each element represents the smallest feature that can be generated by our fabrication method; for multiphoton lithography, these features are defined by the focus region where the light intensity is high enough to initiate resist solidification. The shape of the focus region is well approximated by an axis-aligned ellipsoid and its dimensions have been investigated experimentally [Guney and Fedder 2016]. The size of the focus is mainly influenced by the local dose that is absorbed by the resist. The dose is a function of laser power and laser scan speed, i.e., the brightness and velocity with which an illuminated focus is moved through the resist. Since we keep both parameters fixed during the exposure of a structure, we can assume that the focus ellipsoid has constant size. Thus, we define the 2D profile of a nanostructure as a set $Q$ of elliptic focus regions $Q$ - or short foci - that are parameterized by the 2D location $\mathbf{q}$ of their centers as illustrated in Figure 3.

To simulate the optical behavior of a structure, it is necessary to know the local material parameters throughout the simulation domain. For our purposes, it is sufficient to consider the local permittivity $\epsilon(\mathbf{x})$, which can be obtained from the foci $Q$ as

$$
\epsilon(\mathbf{x})= \begin{cases}\epsilon_{\text {substrate }} & \text { if } y<0 \\ \epsilon_{\text {structure }} & \text { if } y>0 \text { and } \exists Q \in Q \text { with } \mathbf{x} \in Q \\ \epsilon_{\text {air }} & \text { otherwise }\end{cases}
$$

where $\mathbf{x} \in Q$ checks if $\mathbf{x}=(x, y)$ is located inside the focus $Q$ with center $\mathbf{q}=\left(x_{q}, y_{q}\right)$, i.e.,

$$
\left(\frac{x-x_{q}}{w_{Q}}\right)^{2}+\left(\frac{y-y_{q}}{h_{Q}}\right)^{2} \leq \frac{1}{4}
$$

with the constant lateral and vertical focus diameters $w_{Q}$ and $h_{Q}$. We assume that the substrate extends into the lower half space with its surface located at the origin and parallel to the $x$-axis.

\subsection{Constraints}

To ensure that all foci are positioned inside the unit cell, we require that the topmost point of each focus lies between the substrate surface - which is positioned at the origin - and the height of the unit cell above the surface, i.e.,

$$
0 \leq y_{q}+\frac{h_{Q}}{2} \leq H
$$

Foci are allowed to penetrate the substrate to ensure sufficient adhesion of the structure to the substrate. Note that we do not constrain the $x$-components of the foci since we assume periodic boundary conditions. As a shorthand, we denote this constraint as $-h_{Q} / 2 \leq y_{Q} \leq H-h_{Q} / 2$ when applied to each individual focus of a set $Q$ of foci.

\subsection{Structure Optimization}

We formulate the task of finding a structure $Q^{*}$ that realize a userspecified colorization objective $\varphi$ as an optimization problem, i.e.,

$$
Q^{*}=\underset{Q}{\arg \min } \varphi(Q), \quad \text { s.t. } \quad-\frac{h_{Q}}{2} \leq y_{Q} \leq H-\frac{h_{Q}}{2}
$$

which describes a non-linear non-convex bound-constrained problem. Due to the non-convexity, it is hard to obtain a globally optimal solution and we rely on two proven techniques to obtain good approximate solutions: we $i$ ) precompute a dictionary of exemplary structures, which we use to warm-start the search; and ii) we utilize local gradient information to greatly improve the convergence rate to a (local) optimum. Both techniques are essential in our setting, because it is infeasible to rely on random search or arbitrary initializations.

4.4.1 Exemplar Generation. Due to the presence of local minima, it is generally not possible to reach a satisfactory local optimum from every starting configuration. As a remedy, we generate a set $\mathcal{E}$ of exemplary structures and precompute their transmittances. Given a colorization objective $\varphi$, we can evaluate it for each exemplar without any further simulations by directly using Equation (4). The full optimization is performed only for the most promising candidates of $\mathcal{E}$, i.e., those with the smallest objective value.

We generate the exemplars in $\mathcal{E}$ using random sampling. In order to generate structures with varying focus numbers and densities, we first randomly choose a number of foci for each exemplar. For 
this, we specify a range of sensible focus counts in the order of 10 to a few dozens. We employ rejection sampling to obtain the actual structure, i.e., we randomly place the foci in the unit cell and accept the arrangement only if the foci have sufficient pairwise overlap (or sufficient overlap with the substrate). This prevents freefloating foci to be considered as exemplars, since these would not be fabricable. We found that requiring an overlap of at least $30 \%$ of the focus diameter is a good compromise between sampling efficiency and structural stability. This process is repeated until the desired number of exemplars has been generated.

4.4.2 Gradient Direction. The gradient of the objective function $\varphi$ (see Equation (4)) with respect to a focus center $\mathbf{q}$ is given by

$$
\frac{d \varphi}{d \mathbf{q}}=\sum_{L_{\mathrm{i}, j}} \sum_{\breve{\theta}_{k}}(\breve{\mathbf{c}}-\mathbf{c})^{\top} \int_{\lambda} \mathbf{m} \sum_{m} L_{\mathrm{i}, j} \frac{d \tau}{d \mathbf{q}} d \lambda .
$$

By computing the sensitivity of all transmittances $\tau$ with regard to focus movements, we are thus able to determine a focus translation that locally improves the colorization. Performing this computation for all foci, we obtain with $d \varphi / d Q$ the gradient direction for the optimization problem and we use the limited-memory version of the BFGS algorithm with bound constraints (L-BFGS-B) [Byrd et al. 1995] to solve Equation (6).

\section{LIGHT SIMULATION}

In this section, we describe how the two central quantities of our approach - the spectral transmittances $\tau\left(\theta_{\mathrm{i}}, \theta, \lambda, \mathbf{q}\right)$ and their gradient $\frac{d \tau}{d \mathbf{q}}$ with respect to each focus center $\mathbf{q}-$ are computed. For clarity of presentation, all technical details - including our technical contributions - are restricted to this section; it can be skipped if only an overview of our method is of interest.

In the following, our electromagnetic simulation framework is introduced, which includes the adaption of our setting to a FDTD simulation. Next, we describe our handling of polarizations, polychromatic sources, and a novel spectral reconstruction method to reduce otherwise impractical memory requirements. Finally, the transmittance gradient is computed with the help of the adjoint state method, where we take care to avoid ill-defined gradients that arise at the boundaries between regions of different permittivity.

\subsection{Physical Model}

Since we design structures with features sizes that are comparable to the wavelength of visible light (i.e., $0.4-0.7 \mu \mathrm{m}$ ), several assumptions that are commonly employed for light simulations in the field of computer graphics are invalidated. Conventional ray optics cannot simulate the wave behavior of light; even a scalar wave optics approximation cannot capture polarization-dependent effects of wavelength-sized structures [Saleh and Teich 2001, chapter 5.4B]. Thus, we employ the full electromagnetic description of light for our simulation, which is given by the Maxwell equations

$$
\begin{gathered}
\boldsymbol{\nabla} \times \mathcal{E}(\mathbf{x}, t)=-\mu_{0} \frac{\partial \mathcal{H}(\mathbf{x}, t)}{\partial t}-\mathcal{J}_{H}(\mathbf{x}, t) \\
\boldsymbol{\nabla} \times \mathcal{H}(\mathbf{x}, t)=\epsilon(\mathbf{x}) \frac{\partial \mathcal{E}(\mathbf{x}, t)}{\partial t}+\mathcal{J}_{E}(\mathbf{x}, t),
\end{gathered}
$$

where the electric and magnetic field $\mathcal{E}$ and $\mathcal{H}$ are generated by electric and magnetic current sources $\mathcal{J}_{E}$ and $\mathcal{J}_{H}$ - all of which potentially vary in space $\mathbf{x}$ and time $t$. Due to the comparably low light intensities of everyday viewing conditions, materials respond linearly to the fields (i.e., $\mathcal{D}=\epsilon \mathcal{E}$ and $\mathcal{H}=\mu^{-1} \mathcal{B}$ ). Since our setting only consists of dielectric materials (see Equation (5)), we characterize them as lossless (i.e., $\sigma=0$ ) and non-magnetic (i.e., $\left.\mu=\mu_{0}\right)$. Furthermore, we neglect dispersive effects because the refractive indices of our materials vary by not more than $1 \%$ over the visible spectrum [Digaum 2016, 3.2].

For our extruded structures, it is sufficient to simulate the optical behavior of their cross-sectional profiles. In such a 2D setting (i.e., $\partial \cdot / \partial z=0$ ), Equation (7) decouples into two independent parts:

- An out-of-plane $E_{z}$ component with $E_{x}=E_{y}=H_{z}=0$. Referred to as S (orthogonal, German: senkrecht) or TE (transverse electric) polarization.

- An out-of-plane $H_{z}$ component with $H_{x}=H_{y}=E_{z}=0$. Referred to as P (parallel) or TM (transverse magnetic) polarization.

Note that there exists a different nomenclature for polarizations that has 'TE' and 'TM' interchanged since it assumes a waveguide's orientation to be along the $z$-axis, which alters the meaning of 'transverse'. We do not use this alternative definition.

Both polarization settings can be simulated side by side in the same simulation because they act independently of each other. Averaging the results of both yields a nanostructure's optical behavior under unpolarized incident illumination.

As a consequence of linear material responses, the superposition principle holds for Equation (7) and we can regard fields $\mathcal{E}$ and $\mathcal{H}$ as sums of monochrome single-frequency components. For such a component with frequency $f$, the Maxwell equations are given as

$$
\begin{aligned}
\boldsymbol{\nabla} \times \mathbf{E}(\mathbf{x}, f) & =-i \omega \mu_{0} \mathbf{H}(\mathbf{x}, f)-\mathbf{J}_{H}(\mathbf{x}, f) \\
\boldsymbol{\nabla} \times \mathbf{H}(\mathbf{x}, f) & =i \omega \epsilon(\mathbf{x}) \mathbf{E}(\mathbf{x}, f)+\mathbf{J}_{E}(\mathbf{x}, f),
\end{aligned}
$$

with complex phasor quantities $\mathbf{E}, \mathbf{H}, \mathbf{J}_{E}$, and $\mathbf{J}_{H}$. Encoding both local amplitude and phase, a phasor is given as $\mathcal{E}(\mathbf{x}, t)=\mathfrak{R}\left(\mathbf{E}(\mathbf{x}, f) e^{-i \omega t}\right)$ with $\omega=2 \pi f$. We exploit this fact by simulating only those frequencies that are needed for accurate color prediction.

5.1.1 Far Field. In source-free regions with constant permittivity (i.e., $\mathbf{J}_{E}=\mathbf{J}_{H}=\partial \epsilon / \partial \mathbf{x}=0$ ), the Maxwell equations simplify to a wave equation, for which analytic solutions exist. As a consequence, we only need to simulate the full form of Equation (8) in the vicinity of the nanostructure. To obtain the fields at a far-away observer location - with only air in between -, we employ a near-to-farfield transform (NTFFT), which amounts to a spatial inverse Fourier transform $\mathcal{F}^{-1}$ of the near-field quantities $E_{z}$ and $H_{z}$, i.e.,

$$
\begin{aligned}
& E_{z, \mathrm{ff}}\left(k_{x}, f\right)=\frac{e^{i k \rho}}{\sqrt{\rho}} \gamma k_{y}\left(\mathcal{F}^{-1} E_{z}(\cdot, f)\right)\left(\frac{k_{x}}{2 \pi}\right) \\
& H_{z, \mathrm{ff}}\left(k_{x}, f\right)=\frac{e^{i k \rho}}{\sqrt{\rho}} \gamma k_{y}\left(\mathcal{F}^{-1} H_{z}(\cdot, f)\right)\left(\frac{k_{x}}{2 \pi}\right),
\end{aligned}
$$

where $k_{x}=2 \pi\left(m / L+\sin \left(\theta_{\mathrm{i}}\right) / \lambda\right)$ is the $x$-component of the wave vector associated with incident light direction $\theta_{\mathrm{i}}$. Here $m \in \mathbb{Z}$ indexes the permissible discrete outgoing directions; as described in the supplemental material, $m$ coincides with the diffraction orders 
of a grating with period $L$. The wavenumber $k=2 \pi / \lambda$ determines the length of the wave vector and we get $k_{y}=\sqrt{k^{2}-k_{x}^{2}}$, which is real for

$$
\left\lceil-\frac{L}{\lambda}\left(1+\sin \left(\theta_{\mathrm{i}}\right)\right)\right] \leq m \leq\left\lfloor\frac{L}{\lambda}\left(1-\sin \left(\theta_{\mathrm{i}}\right)\right)\right\rfloor
$$

Otherwise, $k_{y}$ is imaginary and waves propagating into the corresponding outgoing directions $\theta=\arcsin \left(k_{x} / \lambda\right)$ are evanescent and decay exponentially in the $y$-direction. We ignore these directions and perform a normalization to ensure that the total near-field energy is transmitted into the non-evanescent directions. See the next Section 5.1.2 for details.

Several quantities, such as $\lambda$ and $k$ - as well as $\epsilon$ and $\mu$ - depend on the material that is present in the far field, which is air in the standard setting and a glass substrate in the reciprocal setting. The observer distance is given by $\rho$ and $\gamma=2 \sqrt{\frac{i}{8 \pi k}}$. Note that arguments $E_{z}$ and $H_{z}$ of the NTFFT are defined on a plane below or above the structure and are tiled in the lateral directions to conform to the unitcell tiling as shown in Figure 3. This effectively turns the Fourier transform into a Fourier series and permits us to directly use the discrete Fourier transform (DFT) when implementing the NTFFT.

5.1.2 Intensity. Given the fields $E_{z \text {,ff }}$ and $H_{z \text {,ff }}$ in the far field, we can compute the associated transmittance $\tau$ by computing the intensity (or spectral irradiance) of the light propagating in this direction. For general fields, the local intensity $I(\mathbf{x}, t)$ at a given time $t$ is given as the magnitude of the Poynting vector $\mathcal{S}(\mathrm{x}, t)=$ $\mathcal{E}(\mathrm{x}, t) \times \mathcal{H}(\mathrm{x}, t)$. In frequency domain, we are interested in the time-averaged intensity $\langle\mathrm{S}\rangle$, i.e.,

$$
I(\mathbf{x}, f)=\langle\mathrm{S}\rangle(\mathbf{x}, f)=\frac{1}{2} \mathfrak{R}(\mathbf{E}(\mathbf{x}, f) \times \overline{\mathbf{H}(\mathbf{x}, f)}) .
$$

For a field along an outgoing direction, it gives

$$
I_{\mathrm{ff}}\left(k_{x}, f\right)=\frac{1}{2 \eta}\left|E_{z, \mathrm{ff}}\left(k_{x}, f\right)\right|^{2} \quad \text { or } \quad I_{\mathrm{ff}}\left(k_{x}, f\right)=\frac{\eta}{2}\left|H_{z, \mathrm{ff}}\left(k_{x}, f\right)\right|^{2} .
$$

In order to ensure that the total intensity that is radiated into the far field is found in the non-evanescent directions, we perform a normalization of the NTFFT. The total far-field intensity is given by $I_{\mathrm{ff}, \text { total }}(f)=\sum_{k_{x}} I_{\mathrm{ff}}\left(k_{x}, f\right)$ where $k_{x}$ is summed over non-evanescent orders according to Equation (10). The total intensity $I_{\text {nf, total }}(f)$ of the near field can be obtained by integrating the Poynting vector over the near-field plane in a unit cell; see the supplemental for details.

The normalized far-field intensity $I_{\text {nff }}$ is given as

$$
I_{\text {nff }}\left(k_{x}, f\right)=I_{\mathrm{fff}}\left(k_{x}, f\right) \frac{I_{\mathrm{nf}, \text { total }}(f)}{I_{\mathrm{ff}, \text { total }}(f)}
$$

and it is used to compute the transmittance $\tau$. The far-field distance terms $\rho$ in Equation (9) are canceled out due to the normalization.

In the reciprocal setting, where light propagates into the glass substrate, we additionally have to account for the glass-air interface at the bottom of the substrate. This is achieved by multiplying $I_{\text {nff }}$ with the appropriate Fresnel transmittance for $\mathrm{S}$ or $\mathrm{P}$ polarization. Note that while $k_{x}$ stays constant across the interface, the wavelength $\lambda$ changes, which also alters $k$ and $k_{y}$. The resulting angular deviation of the light beam matches Snell's law of refraction.

\subsection{FDTD Method}

In the vicinity of a nanostructure, we simulate its interaction with incident light using the finite-difference time-domain (FDTD) method - a proven technique for electromagnetic simulations. It discretizes electric and magnetic field components onto two spatially and temporally staggered grids as shown on the right and it utilizes central finite differences to obtain spatial and temporal derivatives. The system is then evolved in leapfrog fashion by advancing alternately the electric or magnetic fields by one time step using the other field. In the following sections, we first introduce the adaptation of the FDTD

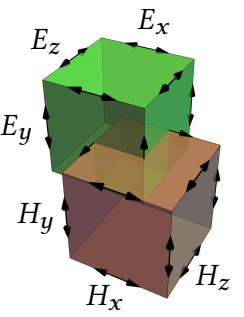
method to our problem and subsequently discuss considerations regarding the light source definition as well as a novel method to reconstruct the output signal from a minimal amount of samples.

5.2.1 Adaptation. The most fundamental parameters for FDTD are the spatial and temporal grid spacings $\Delta \mathbf{x}$ and $\Delta t$. A general recommendation is to have at least 8 spatial samples for each wavelength that occur [Oskooi et al. 2010]. Thus, we set

$$
\Delta \mathbf{x}=\frac{\lambda_{\min }}{8}=\frac{\lambda_{0, \min }}{8 n_{\max }} \quad \text { and } \quad \Delta t=\frac{1}{2} \frac{\Delta \mathbf{x}}{c}
$$

where $\lambda_{0 \text {, min }}$ and $n_{\max }$ are the smallest vacuum wavelength and the largest refractive index of our scene. We choose a Courant factor of $1 / 2$ - well below the Courant-Friedrichs-Lewy (CFL) condition of $n_{\min } / \sqrt{2}=1 / \sqrt{2}$ for our 2D setting - for added numerical stability.

As illustrated in Figure 4, the simulation domain $\Omega$ consists of the aforementioned unit cell $\Omega_{\mathrm{U}}$ (see Figure 3) sandwiched between the source and monitor regions $\Omega_{\mathrm{S}}$ as well as absorbing layers $\Omega_{\mathrm{PML}}$ - all of which are isotropically sampled with spacing $\Delta \mathbf{x}$. The foci arrangement of our structure is then discretized onto the resulting grid inside the unit cell. We avoid discretization artifacts at the boundaries between regions of different permittivity by using the subpixel smoothing technique by Farjadpour et al. [2006].

To realize the lateral tiling of the unit cell, periodic boundary conditions are used in the $x$-direction. Since we assume source-less regions of uniform permittivity above and below the nanostructure, all light should 'disappear' at the boundaries in the $y$-direction. This can be realized with perfectly matched layers (PMLs), which constitute layers of an artificial absorbing material [Berenger 1994]. We employ the uniaxial variant [Taflove and Hagness 2005, ch. 7].

5.2.2 Source Definition. We inject light into our simulation by specifying time-varying electric and/or magnetic source currents $\mathbf{J}_{E}$ or $\mathrm{J}_{H}$ along planar surfaces above and below the unit cell (see $\Omega_{\mathrm{S}}$ in Figure 4). These mimic linearly polarized plane waves that pass through the source region. Since the superposition principle holds in our setting, we spatially overlay currents of different wavelengths and work with polychromatic sources. Here, we account for FDTD-specific issues, such as numerical grid dispersion and PML reflections as described in the supplemental material. As explained in Section 4.1, we operate in a reciprocal setting. Consequently, we inject - during each forward simulation - light from an outgoing direction which then propagates through the nanostructure into 


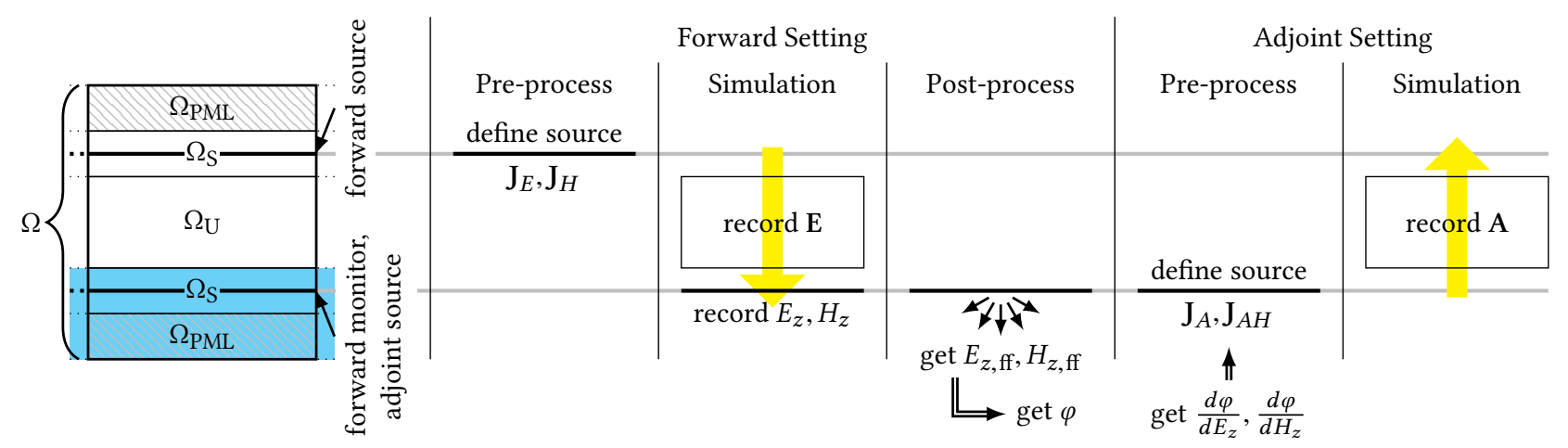

Fig. 4. Simulation Setting. The unit cell $\Omega_{U}$ (see Figure 3) is sandwiched between the source regions $\Omega_{\mathrm{S}}$ and the absorbing layers $\Omega_{\mathrm{PML}}$. The objective function $\varphi$ is computed by $i$ ) defining the (oblique) polychromatic plane wave sources; ii) performing the FDTD simulation and recording near fields $E_{z}$ and/or $H_{z}$ at the monitor plane; iii) computing the far fields $E_{z, \text { ff }}$ and/or $H_{z, \text { ff }}$ to obtain the transmittances; and $i v$ ) performing a color conversion in a post-process. As given in Equation (12), the derivative $d \varphi / d \epsilon$ of the objective with respect to the permittivity distribution $\epsilon$ inside the unit cell is obtained by recording the electric field $\mathbf{E}$ during the forward simulation and additional adjoint simulations. Each adjoint simulation consists of $i$ ) computing the adjoint source current $\mathbf{J}_{A}$ and/or $\mathrm{J}_{A H}$ from the post-processing derivative $d \varphi / d E_{z}$ and/or $d \varphi / d H_{z}$; and ii) performing the FDTD simulation to record the adjoint field $\mathrm{A}$ inside the unit cell.

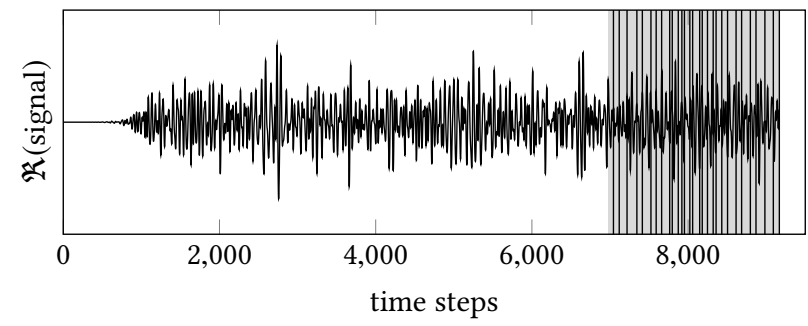

Fig. 5. Signal reconstruction. After transient effects have decayed, the signal needs to be simulated for a sufficient number of time steps to permit decomposition of the signal into its $N_{f}$-many frequency components. Instead of having to record the signal during the whole time interval (gray shaded area), our reconstruction method permits the accurate recovery of the frequency components from only $N_{f}$-many time samples (vertical lines).

the far field below the structure; for each adjoint simulation, light is injected from below (see Figure 4).

5.2.3 Intensity Interpolation. In order to compute the objective function $\varphi$ in the reciprocal setting, we require the knowledge of the outgoing spectral radiance $L\left(\breve{\theta}_{k}, \lambda\right)$ for a set of outgoing directions $\breve{\theta}_{k}$ along which we compute the colors (see Equation (4)). However, due to the aforementioned numerical grid dispersion, it is not possible to propagate all light frequencies in the same direction when simulating them simultaneously in one FDTD run. We solve this issue by interpolating the spectral radiances of the simulated propagation directions. Details on our interpolation scheme can be found in the supplemental material.

5.2.4 Signal Reconstruction. In order to record fields with sufficient accuracy in and around the unit cell, we take two preliminary measures: $i$ ) we wait a sufficient amount of time (i.e., 100 periods of the smallest frequency) to ensure that transient behavior has decayed; and ii) we interpolate the grid values of the magnetic field
- in case they are needed - at the electric grid locations as illustrated at the beginning of Section 5.2.

We are free to record the time-varying complex fields $\mathbf{E}(\mathbf{x}, t)$ and/or $\mathbf{H}(\mathbf{x}, t)$ at any location $\mathbf{x}$ in the simulation domain. Since we use polychromatic sources, these fields are the superposition of the $N_{f}$-many single-frequency components.

For the subsequent near-to-far-field transform (NTFFT), however, we need to reconstruct the individual per-frequency phasor quantities from these superposed fields. Often some form of spectral transform, such as the discrete Fourier transform (DFT) is utilized for this purpose, where the characteristics of the recorded signal (i.e., its temporal resolution and its recording time) determine the resolution in the frequency domain. In our case, however, we know exactly which frequency components are present in the signal, and we can employ a more tailored approach. This avoids any spectral leakage issues that would occur with DFTs - and their sparse variants - since their frequency components hardly ever coincide with the exact components of our signals.

As described in Appendix A, we achieve a reconstruction from only $N_{f}$-many samples of the time signal as shown in Figure 5. Furthermore, we minimize the required simulation time by shrinking the signal recording time window as far as possible without compromising the robustness of the reconstruction.

\subsection{Gradient Computation}

In order to improve the convergence rate of our optimization method, we compute the local gradient of the objective function $\varphi$ with respect to the parameterization of the structure, which is given by the focus centers q. Utilizing Wirtinger calculus [Wirtinger 1927] we can write the objective function's dependency on the complex near-field phasors $\mathrm{E}$ - situated on the monitor plane right below the structure - as $d \varphi=\left(\frac{\partial \varphi}{\partial \mathrm{E}} d \mathrm{E}+\frac{\partial \varphi}{\partial \overline{\mathrm{E}}} d \overline{\mathrm{E}}\right)=2 \mathfrak{R}\left(\frac{\partial \varphi}{\partial \mathrm{E}} d \mathrm{E}\right)$. The full 
gradient is given as

$$
\begin{aligned}
& \text { post-processing derivative parameterization derivative }
\end{aligned}
$$

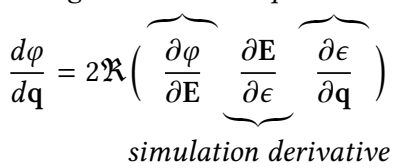

where $\partial \epsilon / \partial \mathbf{q}$ describes how translations of the focus centers $\mathbf{q}$ influence their permittivity discretization $\epsilon$ on the FDTD grid (see Section 5.2.1). $\partial \varphi / \partial \mathrm{E}$ gives the sensitivity of the objective function with respect to the near field; this includes the NTFFT and color conversion and can be easily computed with automatic differentiation [Rall 1981]. The simulation derivative $\partial \mathbf{E} / \partial \epsilon$ denotes the sensitivity of the near fields with regard to changes in the permittivity distribution $\epsilon$. Here, $\mathrm{E}$ acts as a placeholder for both electric and magnetic fields. In the remainder of this section, we show how to efficiently compute the full gradient, how to handle the permittivity discontinuities at the structure boundaries, and how the parameterization gradient is calculated.

5.3.1 Adjoint State Method. Generally, the explicit computation of $\partial \mathrm{E} / \partial \epsilon$ is not feasible due to its vast size of (spatial grid points) $)^{2} \approx$ $10^{9}$ matrix elements. By exploiting the fact that our optimization problem is effectively constrained by the Maxwell equations, i.e., $\mathcal{C}=\left(-\boldsymbol{\nabla} \times \boldsymbol{\nabla} \times+\omega^{2} \mu_{0} \epsilon\right) \mathbf{E}-i \omega \mu_{0} \mathbf{J}_{E}=\mathcal{M} \mathbf{E}-i \omega \mu_{0} \mathbf{J}_{E}=\mathbf{0}$, we can compute $d \varphi / d \mathbf{q}$ with just a single additional simulation as illustrated in Figure 4.

From the constraint derivative

$$
\frac{d C}{d \epsilon}=\frac{\partial \mathcal{C}}{\partial \mathbf{E}} \frac{\partial \mathbf{E}}{\partial \epsilon}+\frac{\partial \mathcal{C}}{\partial \epsilon}=\mathcal{M} \frac{\partial \mathbf{E}}{\partial \epsilon}+\omega^{2} \mu_{0} \mathbf{E}=0
$$

we get

$$
\frac{\partial \varphi}{\partial \mathbf{E}} \frac{\partial \mathbf{E}}{\partial \epsilon}=-\omega^{2} \mu_{0} \frac{\partial \varphi}{\partial \mathbf{E}} \mathcal{M}^{-1} \mathbf{E} \stackrel{!}{=}-\omega^{2} \mu_{0} \mathbf{A}^{\top} \mathbf{E},
$$

where we define the solution $\mathbf{A}$ of $\mathcal{M}^{\top} \mathbf{A}=(\partial \varphi / \partial \mathrm{E})^{\top}$ as the adjoint field, which gives the adjoint state method its name [Lions 1971]. Since differential operators with constant coefficients - such as $\mathcal{M}$ are self-adjoint, we can obtain A with the same simulation method as the forward problem, i.e.,

$$
\mathcal{M A}-i \omega \mu_{0} \mathbf{J}_{A}=0 \quad \text { with } \quad \mathbf{J}_{A}(\mathbf{x}, f)=\frac{1}{i \omega \mu_{0}}\left(\frac{\partial \varphi}{\partial \mathbf{E}}\right)^{\top}(\mathbf{x}, f) .
$$

Since $\partial \varphi / \partial \mathbf{E}$ is non-zero only at the plane where the near field is recorded, the adjoint source current $\mathbf{J}_{A}$ vanishes outside of this area.

5.3.2 Permittivity Discontinuity. By combining Equations (11) and (12) we obtain the full gradient as

$$
\frac{d \varphi}{d \mathbf{q}}=-2 \omega^{2} \mu_{0} \mathfrak{R}\left(\mathbf{A}^{\top} \mathbf{E} \frac{\partial \epsilon}{\partial \mathbf{q}}\right),
$$

which is ill-defined, though. This stems from the fact that electromagnetic fields are generally not continuous across boundaries between regions with different permittivities and this is exactly where $\partial \epsilon / \partial \mathbf{q}$ has its support.

From the integral formulation of the Maxwell equations, one can derive, however, that $\mathbf{n} \times \mathbf{E}$ and $\mathbf{n} \cdot(\epsilon \mathbf{E})$ are continuous across such a boundary with normal vector $\mathbf{n}$. Based on this observation, Johnson et al. [2002] developed an anisotropic smoothing scheme that yields a well-defined gradient expression as a limit:

$$
\begin{aligned}
\frac{d \varphi}{d \mathbf{q}}=-2 \omega^{2} \mu_{0} \int \mathfrak{R}\left(\mathbf{A}_{\|}^{\top} \mathbf{E}_{\|}\left(\epsilon_{\text {structure }}-\epsilon_{\text {air }}\right)\right. \\
\left.+\epsilon^{2} \mathbf{A}_{\perp}^{\top} \mathbf{E}_{\perp}\left(\frac{1}{\epsilon_{\text {air }}}-\frac{1}{\epsilon_{\text {structure }}}\right)\right) \frac{d h}{d \mathbf{q}} \delta(\partial Q) d r
\end{aligned}
$$

where the fields $\mathbf{E}$ and $\mathbf{A}$ are decomposed into parts perpendicular to the local boundary (i.e., $\epsilon \mathbf{E}_{\perp}=\epsilon(\mathbf{E} \cdot \mathbf{n}) \mathbf{n}$ ) and parallel to it (i.e., $\left.\mathbf{E}_{\|}=\mathbf{E}-\mathbf{E}_{\perp}\right)$, which are both continuous across the boundary. $d h / d \mathbf{q}$ denotes the changes in local surface height with respect to translations of the focus center $q$. These changes are accumulated along the focus boundary $\partial Q$ using a Dirac delta distribution $\delta$.

5.3.3 Parameterization Gradient. Moving the center q of a focus $Q$ causes a translation of the whole focus. The resulting change in surface height $h$ around its perimeter is simply $d h=\mathbf{n}_{Q} \cdot d \mathbf{q}$ where $\mathbf{n}_{Q}$ denotes the local surface normal of the focus ellipse. Due to potential overlaps with the substrate or other foci, there might be sections along the focus perimeter $\partial Q$ where a focus shift does not result in a change of the local permittivity as shown on the right. The movement of a focus that lies completely inside the substrate - to give an extreme example - should not influence the objective function in any way. Consequently, only those parts $\widetilde{\partial Q}$ of a focus boundary $\partial Q$, which are in contact

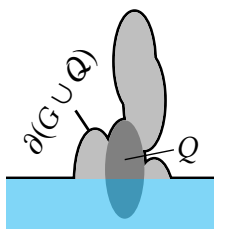
with air, should be considered in Equation (13). Formally, we have $\widetilde{\partial Q}=\partial Q \cap \partial(G \cup Q)$, with the glass substrate $G$ and the set of all foci $Q$. This yields the final expression of the gradient

$$
\begin{aligned}
\frac{d \varphi}{d \mathbf{q}}=-2 \omega^{2} \mu_{0} \int_{\widetilde{\partial Q}} & \mathfrak{R}\left(\mathbf{A}_{\|}^{\top} \mathbf{E}_{\|}\left(\epsilon_{\text {structure }}-\epsilon_{\text {air }}\right)\right. \\
& \left.+\epsilon^{2} \mathbf{A}_{\perp}^{\top} \mathbf{E}_{\perp}\left(\frac{1}{\epsilon_{\text {air }}}-\frac{1}{\epsilon_{\text {structure }}}\right)\right) \mathbf{n}_{Q} d r .
\end{aligned}
$$

\section{FABRICATION}

As a result of the optimization problem in Equation (6), we obtain a nanostructure that realizes a given colorization objective. The description of the structure already well-suited for our fabrication method and only minor post-processing is required.

\subsection{Structural Reinforcement}

The focus movement during the design optimization could reduce their pairwise overlap to a point where the structural integrity of the design is compromised. As a result, insufficiently overlapping foci could separate from each other (or from the substrate) during the actual fabrication. We avoid this by adding additional foci to the design as a reinforcement - shown as gray regions outside the focus ellipses in Figures 6 and 7.

As a first step, we generate a neighborhood graph between the nearest neighbors. The vertices of the graph are both the foci and the substrate; as a distance measure, we use the distance between 
the focus centers and their vertical distance from the substrate surface. To identify the principal structure of our design, we compute a minimum spanning tree on this graph. Along this tree, we add reinforcement foci to anchor our structure onto the substrate and to ensure sufficient structural stability. For this, we enforce a maximal spacing of $100 \mathrm{~nm}$ in the vertical and $40 \mathrm{~nm}$ in the horizontal direction. As a result, we obtain a reinforced version of our design with only minor changes in its shape.

Alternatively, we could have used such a dense focus arrangement directly in the design optimization and enforce the maximal spacing with additional constraints. Note that this would have no impact on the simulations itself since the structure is discretized onto the FDTD grid. However, this added complexity negatively impacts the convergence rate of the optimization due to the non-linearity of the new constraints. Thus, we forwent such an approach.

\subsection{Blueprint Generation}

The parameterization of our nanostructures in terms of focus centers can be immediately translated into a fabrication blueprint. Each center point is extruded as a line orthogonal to the profile and parallel to the substrate. By choosing the length of the line to be the width $L$ of the unit cell, this extrusion generates a quadratic patch of side length $L$ on the surface. Larger areas of the substrate can be colorized by tiling this patch across the surface. Spatially varying colorizations are supported by altering the structures of the patches.

The commands of the blueprint are ordered in such a way that the lines are generated bottom-up. This ensures that all lines are well anchored onto the substrate; otherwise disconnected lines might float away during the fabrication process due to diffusion or heat convection in the resist. As an additional benefit, this ordering also moves the Piezo table - which controls the focus height - in only one direction, which avoids any hysteresis effects that could occur with ambidirectional Piezo motion.

If the same structure is tiled over a larger area, all lines with the same height are written together. This further minimizes the comparably slow Piezo table movement and maximizes the usage of the fast lateral focus movements through mirror galvanometers. This is possible for patches of up to $50 \mu \mathrm{m} \times 50 \mu \mathrm{m}$ beyond which vignetting and distortion effects appear. To move the focus beyond that region, the motorized stage is used and after each of its use, the device is instructed to calibrate the focus height with respect to the substrate interface again.

\subsection{Realization}

As fabrication method, we employ multiphoton lithography. It uses a two-photon polymerization process to selectively solidify a UVcurable negative-tone photoresist. This is achieved with a nearinfrared ultra-short-pulsed laser that produces high light intensities in a small focus region. Only in this region, the photon density is high enough to make it possible that two of them are absorbed in short succession, i.e., the second photon is absorbed before the excitation of the first absorption is decayed. The energies of this two photons accumulate and pass the polymerization threshold which usually requires UV light. We use the commercially available Photonic Professional GT by Nanoscribe GmbH as fabrication device.
In our case, it was installed in a cleanroom environment, which is not necessarily required.

As photoresist, we utilize IP-Dip by the same company. It is optimized for small-scale dip-in laser lithography (DiLL) - which we employ - where the objective of the fabrication device is submerged in the resist. As substrate we use transparent glass slate made out of fused silica, supplied by Nanoscribe as DiLL substrates. The refractive indices of both the photoresist and the substrate are sufficiently different to allow the device to automatically detect the interface between them for calibration purposes. After exposure, we use a $m r$-Dev 600 developer by Micro Resist Technology GmbH to remove all unpolymerized resist. After the final cleaning with isopropanol (IPA), we use an Automegasamdri 916B Series $C$ critical point dryer by Tousimis Research Corporation to evaporate the IPA without causing capillary forces that could damage our sample. Note that this last step could potentially be replaced by direct evaporation of the IPA or by a final bath in deionized (DI) water and its direct evaporation.

We employed the following process steps:

(1) Rinse substrate with acetone, IPA, and DI water. Blow-dry with nitrogen.

(2) Apply IP-Dip via drop casting and mount sample on holder.

(3) Insert holder into device and expose structure via DiLL, which took $10-20 \mathrm{~h}$ for the samples shown in Figures 9 to 11.

(4) Remove holder from device and develop sample in developer bath for $20 \mathrm{~min}$.

(5) Clean sample in IPA bath for $\geq 5$ min.

(6) Dry sample using the critical point dryer with default settings.

Afterwards, the sample is ready for use.

\section{RESULTS}

In this section, we present various nanostructures that were designed with the help of our method. We show both simulated colorization as well as measurements of fabricated samples.

\subsection{Implementation}

We implemented our design system - including the NTFFT and the adjoint method - largely in Python using SciPy [Jones et al. 01] and NumPy [van der Walt et al. 2011] for all numeric computations including the rank-revealing QR (RRQR) decomposition and L-BFGS-B. The color conversions are based on the python-colormath package by Greg Taylor (see https://pypi.python.org/pypi/colormath/) while automatic differentiation is performed with the autograd package by Dougal Maclaurin, David Duvenaud, and Matthew Johnson (see https://pypi.python.org/pypi/autograd). The FDTD method was implemented using Meep [Oskooi et al. 2010], which provided subpixel smoothing as well as the periodic and PML boundary conditions.

Unless explicitly stated otherwise, we used the following parameters to generate our results: All design optimizations were initialized using a set $\mathcal{E}$ of 1000 exemplars. The unit cell dimensions $L=5 \mu \mathrm{m}$ and $H=2 \mu \mathrm{m}$ and the dimensions of the focus region $w_{Q}=400 \mathrm{~nm}$ and $h_{Q}=1 \mu \mathrm{m}$, whose dependency on our setting for laser power $L P=80 \%$ and scan speed $s s=3000 \mu \mathrm{m} \mathrm{s}^{-1}$ has been thoroughly investigated in previous work [Guney and Fedder 2016]. See the 


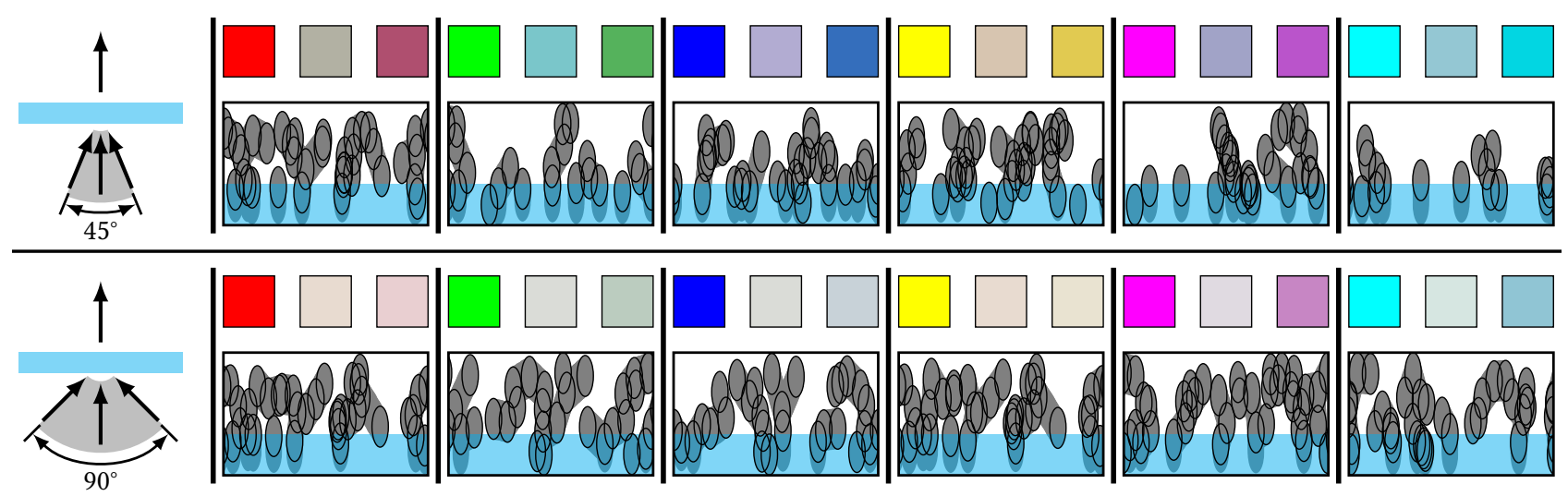

Fig. 6. Optimization results for a colorization of the normal viewing direction with incident $\mathrm{D}_{65}$ daylight from a $45^{\circ}$ cone (top row) and a $90^{\circ}$ cone (bottom row). For each row, the scene setup is illustrated (far left) and six results are shown for the target colors red, green, blue, yellow, magenta, and cyan (left to right) of the sRGB gamut. For each result, one unit cell of the optimized and reinforced nanostructure is depicted. The three color boxes above each cell indicate the target color of the colorization objective (left), the simulated color of the exemplar - with which the optimization is initialized - (center), and the simulated color of the nanostructure below (right).
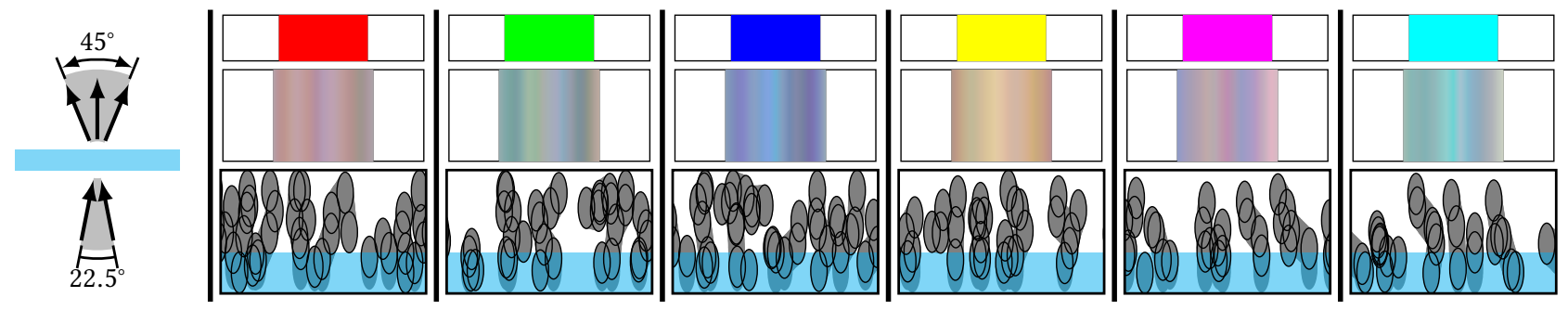

Fig. 7. Optimization results for a colorization of the outgoing viewing directions in a $22.5^{\circ}$ cone with incident $D_{65}$ daylight from a $22.5^{\circ}$ cone. The scene setup is illustrated (far left) and six results are shown for the target colors red, green, blue, yellow, magenta, and cyan (left to right) of the sRGB gamut. For each result, one unit cell of the optimized and reinforced nanostructure is depicted (bottom) as well as its simulated colors (center) and the target colors of the colorization objective (top) in a $45^{\circ}$ cone. The directions outside the target specifications are not simulated and are left white.

Nanoscribe control file blueprint.gwl in the supplemental for details on the construction process of the sample shown in Figure 9. As scene permittivities, we set $\epsilon_{\text {air }}=\epsilon_{0}=1.0, \epsilon_{\text {structure }}=1.54^{2} \epsilon_{0}$, and $\epsilon_{\text {substrate }}=1.46^{2} \epsilon_{0}$, where we obtained the refractive indices of the polymerized resist and the substrate from measurement by Digaum [2016] and the substrate supplier.

We used an Intel ${ }^{\circledR}$ E5-1620 v4 workstation with 32 GB system memory to run our method. The average runtime of each optimization was $(2.3 \pm 1.3) \mathrm{h}$ for the single-viewing-direction results of Figure 6 and $(6.0 \pm 3.6) \mathrm{h}$ for multiple viewing directions as shown in Figures 7 and 8. Detailed statistics are given in the supplemental material. We used different imaging modalities to investigate our structures, such as electron microscopy (Figures 1, 9 and 10), transmission brightfield microscopy (Figures 1 and 12), and macrophotography (Figures 9 to 11 and 13); details can be found in the supplemental material.

\subsection{Optimized Designs}

We used our system to design nanostructures for different illumination conditions and colorization objectives. Due to the limited resolution of direct laser writing (DLW), we expect our structures to exhibit a trade-off between $i$ ) the range of viewing directions over which we want to control the colorization; ii) how close the target colors are reproduced; and iii) the size of the angular range under which illumination is incident on the structure. In Section 7.2.1, we limit the viewing angles to the normal direction and show optimized nanostructures for various target colors and incident illumination. Afterwards, in Section 7.2.2, we show results optimized for multiple viewing directions. Accompanying numerical results in form of color values and color differences can be found in the supplemental.

7.2.1 Normal Viewing Direction. By limiting the target color specification to only the normal viewing directions, we effectively realize color filters for wide-angle illuminations. For the extreme case of only normally incident light, this would match the setting of Nawrot et al. [2013], who fabricated regular pillar arrays. Since we are interested in more realistic lighting conditions, we specified light to be incident from cones with both $45^{\circ}$ and $90^{\circ}$ opening angles as shown in Figure 6 (top and bottom). We observed that for the smaller opening angle, a wide range of colors can be realized. To give an overview of the possible color gamut, we reproduce the 


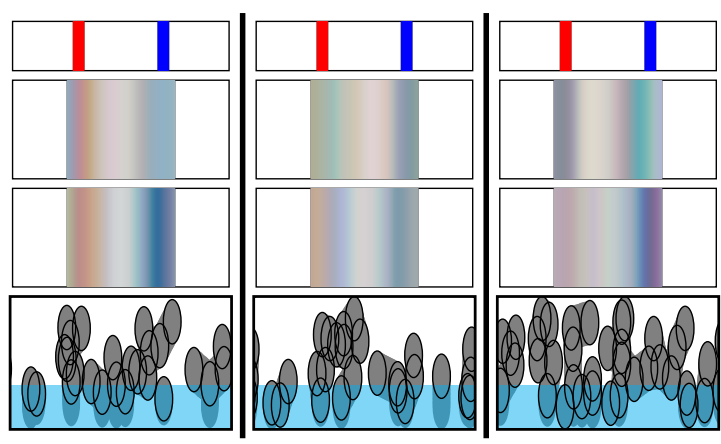

Fig. 8. Optimization results for an asymmetric colorization of the outgoing viewing directions with a scene specification as in Figure 7. Apart from the target colors of the red-blue split (top), the unit cell (bottom), and the optimized colors (center bottom), the simulated colors of the exemplar - with which the optimization is initialized - is shown (center top). The optimization results for three different exemplar initializations are shown.

extremal colors of the sRGB color space as targets, i.e., red, green, blue, yellow, magenta, and cyan.

Doubling the opening angles limits the nanostructures' ability to redirect selected spectral components away from the illuminated region, which, in turn, significantly reduces the color contrast while the color hue of all targets is still maintained. Note that this setting is the mirror image of the setting of Andkjær et al. [2013], who use a single incident light direction but optimize reflected structural color over a wide angular range. Their results indicate that future technological advances of direct laser writing (DLW) in terms of resolution and/or refractive index contrast would significantly improve the color contrast of our result.

A qualitative inspection of the structures shows that there is no immediate intuitive relationship between the geometry of the nanostructure and the resulting color. This makes the manual design of such freeform shapes extremely demanding and computational assistance is indispensable. Furthermore, we can observe that a brute-force search in the design space does not yield a useful nanostructure since we do this already during the exemplar-based initialization of our optimization. As can be seen in the color patches above each structure in Figure 6, the simulated color of the exemplar (center patch) is far from the target color (left patch). Using gradient information during the optimization - as we do by employing the adjoint state method - produces a much closer fit (right patch) of the target color.

7.2.2 Wide-angle Viewing Directions. Designing nanostructures that realize colorization for varying viewing directions can also be achieved with our method. We show results for symmetric color targets in Figure 7 as well as for an asymmetric target in Figure 8 . Using the same color samples as in Figure 6, we can see that our method manages to reproduce the correct hue for all colors although angular variations exist. These are caused by the diffractive nature of our periodic structures. Adding disorder to this otherwise perfect tiling would smear out the colors and improve their stability over the range of viewing directions; see Section 8 for details.
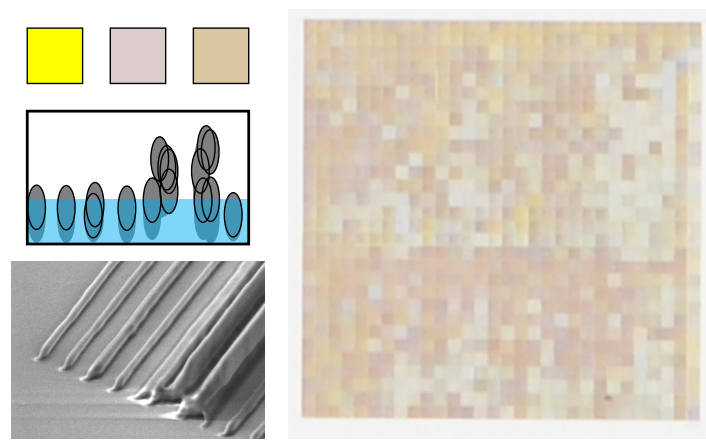

Fig. 9. Fabricated sample optimized for a yellow colorization of the normal viewing direction. A photograph of the $1.5 \mathrm{~mm} \times 1.5 \mathrm{~mm}$ sample is shown (right) as well as a micrograph of the nanostructures of one $5 \mu \mathrm{m}$-long unit cell (bottom left). See Figure 6 for a description of the illustration (top left).
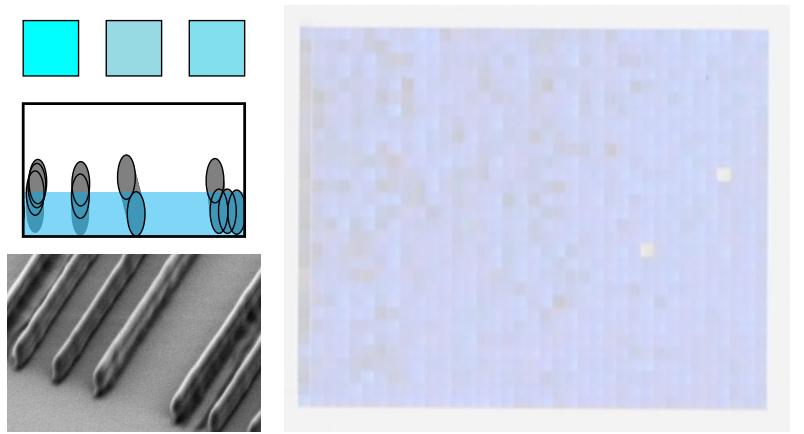

Fig. 10. Fabricated sample optimized for a cyan colorization of the normal viewing direction. A photograph of the $1.85 \mathrm{~mm} \times 1.5 \mathrm{~mm}$ sample is shown (right) as well as a micrograph of the nanostructures of one $5 \mu \mathrm{m}$-long unit cell (bottom left). See Figure 6 for a description of the illustration (top left).

Since the influence of a nanostructure on the incident light varies with the light's wavelength, it is possible to redirect spectral components of the incident light into different directions. As shown in Figure 8, we are able to design structures cause red and blue colorization on different sides of the surface normal.

In the same figure, we also illustrate the effect of using different exemplars to initialize our optimization. By using gradient information, we are able to improve the nanostructures such that the final colorization (third row) matches the color targets (top row) much closer than the initial exemplar colors (second row).

Our general experience is that a better initial guess also leads to a better final result. This indicates that our colorization objective function exhibits many local minima, whose treatment open the avenue for interesting future research. At the same time, very different structure realize the same colorization objective, which indicates a rich design space. For different applications, it seems possible to introduce further constraints to our optimization problem and still obtain useful results. 


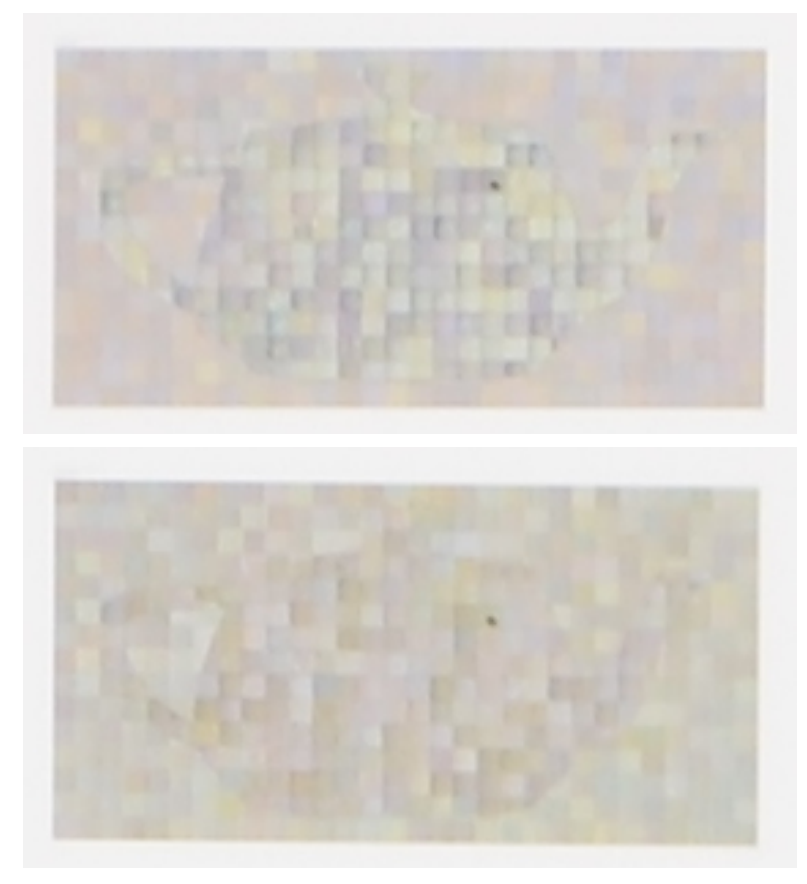

Fig. 11. Fabricated sample optimized for an asymmetric colorization of the background. A photograph of the $1.5 \mathrm{~mm} \times 0.75 \mathrm{~mm}$ sample shows that varying the viewing angle from $10^{\circ}$ (top) to $-10^{\circ}$ (bottom) causes a color shift of the background from blue to red. See Figure 1 for additional details on the sample.

\subsection{Fabricated Samples}

We fabricated several nanostructures that were designed and reinforced with our method using the setup described in Section 6.3. As shown in Figures 9 and 10, colorization of the normal viewing direction could be achieved for opposing colors (i.e., yellow and blue). In Figures 1 and 11, spatially varying optical behavior across the sample is shown by assigning different optimized nanostructures to the background and the teapot-shaped foreground. The background was designed to exhibit a blue-red color split similar to Figure 8, which can be observed under wide-angle illumination as shown in Figure 11. The foreground was assigned a stable green color.

Our structures show superior angular colorization stability when compared with simple phase grating structures [Nawrot et al. 2013] that were shown to work well for normally incident light (see Figure 13). To match our 2D setting, we converted the original structure of Nawrot et al., which consisted of regularly spaced pillars of uniform height, to parallel 'walls' of uniform height. The colorization effect of these structures depend on their height and - to a lesser degree - on the wall spacing. Their angular stability is similar across their color gamut as is shown in Figure 13 (top row), where the four patches were fabricated with $1.2 \mu \mathrm{m}$ wall spacing and wall heights of 1.1, 1.4, 1.7, and $2.0 \mu \mathrm{m}$.

For our fabricated samples, the angular viewing range under which the colorization is preserved is up to 2.5 times larger (i.e., $\approx 40^{\circ}$ instead of $\approx 15^{\circ}$ ). Since transparent nanostructures redirect certain spectral components of the incident light away from the direction
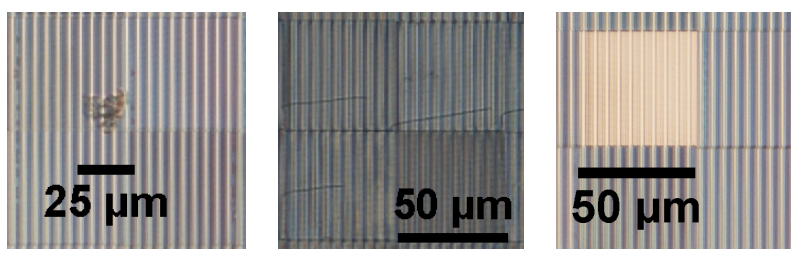

Fig. 12. Possible failure modes of direct laser writing (DLW). Airborne pollutants such as dust particles cause local structure damage (left). Insufficient structure reinforcement leads to a detachment of one or more lines during development (center). Imprecise detection of the resist-substrate interface results in diminished or exaggerated structure heights (right).

where they are unwanted, we expect them to show up at different viewing directions. Indeed, we can observe a color inversion for oblique viewing angles where the colors of our fabricated samples effectively switch as shown in Figure 13 (first and last column). Simple phase gratings are less stable against this effect as well.

\section{LIMITATIONS AND FUTURE WORK}

We encountered an apparent fabrication deficiency with our fabrication device (i.e., a Photonic Professional GT) that is not necessarily related to using DLW in general. All our fabricated examples consist of small $50 \mu \mathrm{m} \times 50 \mu \mathrm{m}$ patches as described in Section 6.2. While the colorization is uniform inside each patch, the colors between different patches may vary greatly although all their blueprints are identical. Investigations with electron microscopy revealed that although the structures of each patch had similar geometry, their location relative to the substrate varied; see Figure 12 (right) for an extreme case. This indicates that the interface finder, which determines the location of the substrate surface, is inaccurate and - because we calibrate the fabrication height for each patch independently this height inaccuracy manifest as varying colors throughout our samples. Figure 12 list further fabrication issues.

We consider this work a first step in the computational freeform design of structural colorizations and there are multiple avenues for future research. Adding a certain degree of disorder to the nanostructures would reduce diffraction effects [Ghiradella 1991] with one example being height randomizations [Johansen 2014]. However, printing thin pedestals with DLW is prone to deformation due to shrinkage [Bauhofer et al. 2017]. Nonuniform line shapes could be achieved by varying the laser power and/or the scan speed across the structure, however extensive device calibrations would be needed. General 3D structures are inherently supported by DLW but a full 3D FDTD-based optimization is highly resource intensive and a more tailored approach would be required. Using related nanofabrication methods such as stimulated emission depletion (STED) [Wollhofen et al. 2013] or nanoimprint lithography would allow smaller feature size or faster fabrication. Altering the structure material, adding pigmentations, or using reflective substrates could expand the range of optical effects as well.

\section{CONCLUSIONS}

In this work, we combined computational design methods with additive manufacturing at the nanoscale to create structural colorizations 


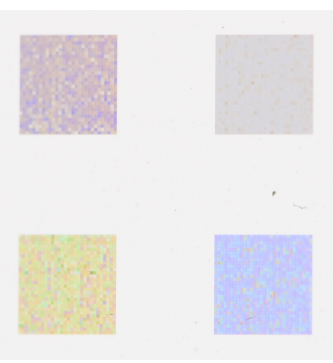

$15^{\circ}$

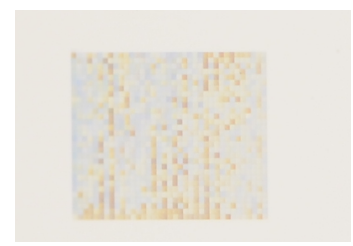

$20^{\circ}$

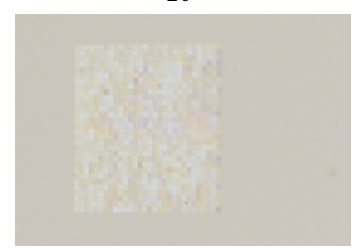

$30^{\circ}$

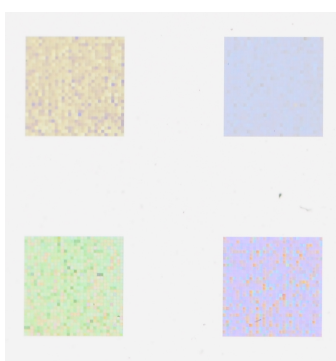

$7.5^{\circ}$

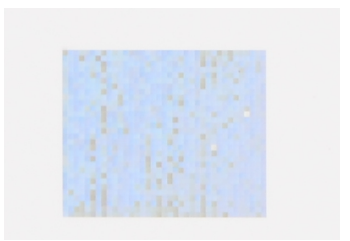

$10^{\circ}$

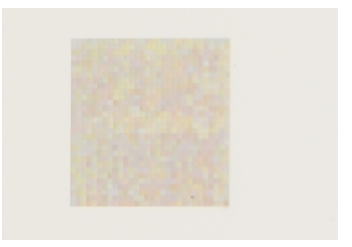

$20^{\circ}$

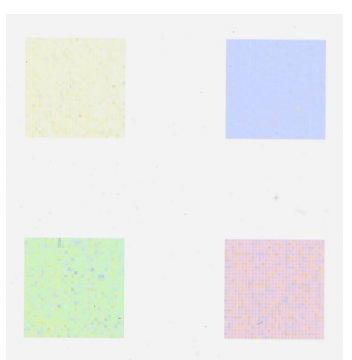

$0^{\circ}$

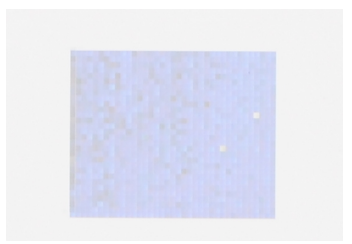

$0^{\circ}$

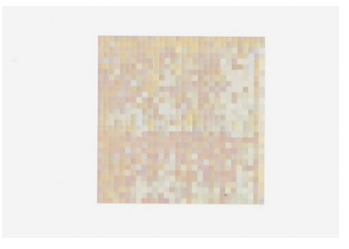

$0^{\circ}$

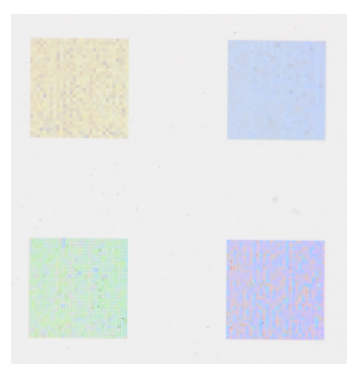

$-7.5^{\circ}$

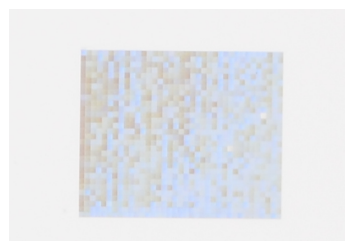

$-10^{\circ}$

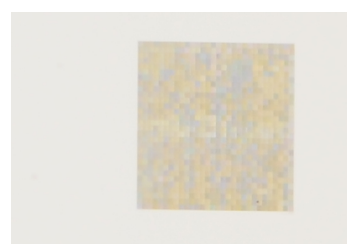

$-20^{\circ}$

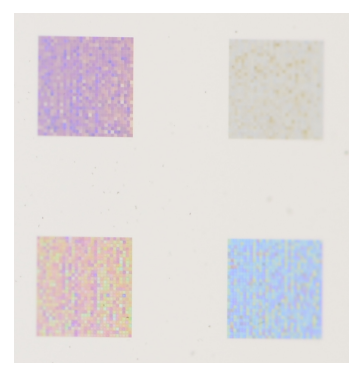

$-15^{\circ}$

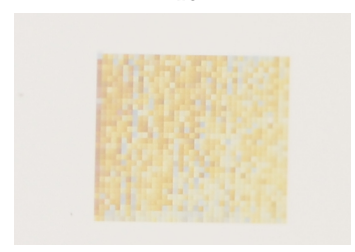

$-20^{\circ}$

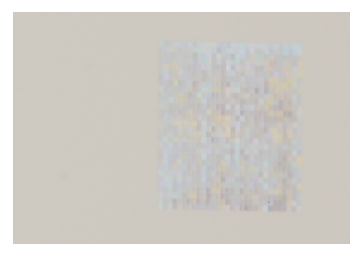

$-30^{\circ}$

Fig. 13. Superior angular stability of our samples (see Figures 9 and 10) when compared to regular phase gratings in the form of parallel fences [Nawrot et al. 2013] (top row). Apart from the normal viewing direction (center column), the onset angle of discolorizations (center left and right), as well as the onset angle of color inversion (far left and right) is shown. Both our samples (center and bottom row) maintain their intended colorization up to higher viewing angles than simple grating structures.

of surfaces. We integrated electromagnetic FDTD simulations into a gradient-based optimization framework using the adjoint state method and presented a novel signal reconstruction method to reduce its memory requirements. With a structure parameterization that inherently adheres to fabrication constraints, we are able to design nanostructures that realize a wide range of colorizations under different illumination conditions. We successfully fabricated several structures using DLW and verified their optical behavior with various measurements.

\section{ACKNOWLEDGMENTS}

We would like to thank Zechen Zhang, Steve Marschner, and Qiang Fu for insightful discussions. For help with nanofabrication aspects, we want to thank Zhihong Wang, Xiong Dun, Andrea Bertoncini, and - especially - Arnold Priebe. We also want to thank the anonymous referees for their valuable suggestions. Thomas Auzinger and Bernd Bickel are supported by the European Research Council Starting Grants under Grant No.: 715767 (https: //cordis.europa.eu/project/rcn/206323_en.html). This work was in part supported by King Abdullah University of Science and Technology Baseline Funding. The fabrication of the nanostructures was performed in the KAUST Nanofabrication Core Lab.

\section{REFERENCES}

Carlos I. Aguirre, Edilso Reguera, and Andreas Stein. 2010. Colloidal Photonic Crystal Pigments with Low Angle Dependence. ACS Applied Materials \& Interfaces 2, 11 (nov 2010), 3257-3262. https://doi.org/10.1021/am100704f

Jacob Andkjær, Villads Egede Johansen, Kasper Storgaard Friis, and Ole Sigmund. 2013. Inverse design of nanostructured surfaces for color effects. Fournal of the Optical Society of America B 31, 1 (dec 2013), 164. https://doi.org/10.1364/josab.31.000164

Vahid Babaei, Kiril Vidimče, Michael Foshey, Alexandre Kaspar, Piotr Didyk, and Wojciech Matusik. 2017. Color contoning for 3D printing. ACM Transactions on Graphics 36, 4 (jul 2017), 1-15. https://doi.org/10.1145/3072959.3073605

Anton A. Bauhofer, Sebastian Krödel, Jan Rys, Osama R. Bilal, Andrei Constantinescu, and Chiara Daraio. 2017. Harnessing Photochemical Shrinkage in Direct Laser Writing for Shape Morphing of Polymer Sheets. Advanced Materials 29, 42 (sep 2017), 1703024. https://doi.org/10.1002/adma.201703024

Fermín S. V. Bazán. 2000. Conditioning of Rectangular Vandermonde Matrices with Nodes in the Unit Disk. SIAM 7. Matrix Anal. Appl. 21, 2 (jan 2000), 679-693. https://doi.org/10.1137/s0895479898336021

Laurent Belcour and Pascal Barla. 2017. A practical extension to microfacet theory for the modeling of varying iridescence. ACM Transactions on Graphics 36, 4 (jul 2017), 1-14. https://doi.org/10.1145/3072959.3073620

Jean-Pierre Berenger. 1994. A perfectly matched layer for the absorption of electromagnetic waves. F. Comput. Phys. 114, 2 (oct 1994), 185-200. https://doi.org/10.1006/ jcph.1994.1159

Amit H. Bermano, Thomas Funkhouser, and Szymon Rusinkiewicz. 2017. State of the Art in Methods and Representations for Fabrication-Aware Design. Computer Graphics Forum 36, 2 (may 2017), 509-535. https://doi.org/10.1111/cgf.13146

Alan Brunton, Can Ates Arikan, and Philipp Urban. 2015. Pushing the Limits of 3D Color Printing: Error Diffusion with Translucent Materials. ACM Transactions on Graphics 35, 1 (dec 2015), 1-13. https://doi.org/10.1145/2832905

Ian B. Burgess, Marko Lončar, and Joanna Aizenberg. 2013. Structural colour in colourimetric sensors and indicators. Fournal of Materials Chemistry C 1, 38 (2013), 6075. https://doi.org/10.1039/c3tc30919c 
Richard H. Byrd, Peihuang Lu, Jorge Nocedal, and Ciyou Zhu. 1995. A Limited Memory Algorithm for Bound Constrained Optimization. SIAM fournal on Scientific Computing 16, 5 (sep 1995), 1190-1208. https://doi.org/10.1137/0916069

Jennefir Digaum. 2016. Fabrication and Characterization of Spatially-Variant SelfCollimating Photonic Crystals. phdthesis. University of Central Florida. http: //purl.fcla.edu/fcla/etd/CFE0006527

Yue Dong, Jiaping Wang, Fabio Pellacini, Xin Tong, and Baining Guo. 2010. Fabricating spatially-varying subsurface scattering. ACM Transactions on Graphics 29, 4 (jul 2010), 1. https://doi.org/10.1145/1778765.1778799

Oskar Elek, Denis Sumin, Ran Zhang, Tim Weyrich, Karol Myszkowski, Bernd Bickel, Alexander Wilkie, and Jaroslav Krrivánek. 2017. Scattering-aware texture reproduction for 3D printing. ACM Transactions on Graphics 36, 6 (nov 2017), 1-15. https://doi.org/10.1145/3130800.3130890

Moritz Emons, Kotaro Obata, Thomas Binhammer, Aleksandr Ovsianikov, Boris N Chichkov, and Uwe Morgner. 2012. Two-photon polymerization technique with sub-50 nm resolution by sub-10 fs laser pulses. Optical Materials Express 2, 7 (jun 2012), 942. https://doi.org/10.1364/ome.2.000942

Grant T England and Joanna Aizenberg. 2017. Emerging optical properties from the combination of simple optical effects. Reports on Progress in Physics 81, 1 (nov 2017), 016402. https://doi.org/10.1088/1361-6633/aa8372

A. Farjadpour, David Roundy, Alejandro Rodriguez, M. Ibanescu, Peter Bermel, J. D. Joannopoulos, Steven G. Johnson, and G. W. Burr. 2006. Improving accuracy by subpixel smoothing in the finite-difference time domain. Optics Letters 31, 20 (sep 2006), 2972. https://doi.org/10.1364/ol.31.002972

Yulan Fu, Cary A. Tippets, Eugenii U. Donev, and Rene Lopez. 2016. Structural colors: from natural to artificial systems. Wiley Interdisciplinary Reviews: Nanomedicine and Nanobiotechnology 8, 5 (mar 2016), 758-775. https://doi.org/10.1002/wnan.1396

Henning Galinski, Gael Favraud, Hao Dong, Juan S Totero Gongora, Grégory Favaro, Max Döbeli, Ralph Spolenak, Andrea Fratalocchi, and Federico Capasso. 2016. Scalable, ultra-resistant structural colors based on network metamaterials. Light: Science \& Applications 6, 5 (sep 2016), e16233. https://doi.org/10.1038/lsa.2016.233

Helen Ghiradella. 1991. Light and color on the wing: structural colors in butterflies and moths. Applied Optics 30, 24 (aug 1991), 3492. https://doi.org/10.1364/ao.30.003492

Walton C. Gibson. 2014. The Method of Moments in Electromagnetics (2 ed.). Chapman and Hall/CRC, Boca Raton, FL.

Ming Gu and Stanley C. Eisenstat. 1996. Efficient Algorithms for Computing a Strong Rank-Revealing QR Factorization. SIAM fournal on Scientific Computing 17, 4 (jul 1996), 848-869. https://doi.org/10.1137/0917055

X. Wendy $\mathrm{Gu}$ and Julia. R. Greer. 2015. Ultra-strong architected $\mathrm{Cu}$ meso-lattices Extreme Mechanics Letters 2 (mar 2015), 7-14. https://doi.org/10.1016/j.eml.2015.01. 006

M G Guney and G K Fedder. 2016. Estimation of line dimensions in 3D direct laser writing lithography. Journal of Micromechanics and Microengineering 26, 105011 (sep 2016), 10. https://doi.org/10.1088/0960-1317/26/10/105011

Miloš Hašan, Martin Fuchs, Wojciech Matusik, Hanspeter Pfister, and Szymon Rusinkiewicz. 2010. Physical reproduction of materials with specified subsurface scattering. ACM Transactions on Graphics 29, 4 (jul 2010), 1. https://doi.org/10.1145/1778765. 1778798

Nicolas Holzschuch and Romain Pacanowski. 2017. A two-scale microfacet reflectance model combining reflection and diffraction. ACM Transactions on Graphics 36, 4 (jul 2017), 1-12. https://doi.org/10.1145/3072959.3073621

Chia Wei Hsu, Owen D. Miller, Steven G. Johnson, and Marin Soljačić. 2015. Optimization of sharp and viewing-angle-independent structural color. Optics Express 23, 7 (apr 2015), 9516. https://doi.org/10.1364/oe.23.009516

Matthias B. Hullin, Ivo Ihrke, Wolfgang Heidrich, Tim Weyrich, Gerwin Damberg, and Martin Fuchs. 2013. Computational Fabrication and Display of Material Appearance. In Eurographics 2013 - State of the Art Reports, M. Sbert and L. Szirmay-Kalos (Eds.) The Eurographics Association, 1-17. https://doi.org/10.2312/conf/eg2013/stars/ 137-153

Jian-Ming Jin. 2014. The Finite Element Method in Electromagnetics (3 ed.). John Wiley \& Sons, New York.

Villads Egede Johansen. 2014. Optical role of randomness for structured surfaces. Applied Optics 53, 11 (apr 2014), 2405. https://doi.org/10.1364/ao.53.002405

Villads Egede Johansen, Jacob Andkjær, and Ole Sigmund. 2014. Design of structurally colored surfaces based on scalar diffraction theory. Fournal of the Optical Society of America B 31, 2 (jan 2014), 207. https://doi.org/10.1364/josab.31.000207

Villads Egede Johansen, Lasse Højlund Thamdrup, Kristian Smistrup, Theodor Nielsen, Ole Sigmund, and Peter Vukusic. 2015. Designing visual appearance using a structured surface. Optica 2, 3 (mar 2015), 239. https://doi.org/10.1364/optica.2.000239

Steven G. Johnson, M. Ibanescu, M. A. Skorobogatiy, O. Weisberg, J. D. Joannopoulos, and Y. Fink. 2002. Perturbation theory for Maxwell's equations with shifting materia boundaries. Physical Review E 65, 6 (jun 2002), 7. https://doi.org/10.1103/physreve. 65.066611

Eric Jones, Travis Oliphant, Pearu Peterson, et al. 2001-. SciPy: Open source scientific tools for Python. (2001-). http://www.scipy.org/ [Online; accessed 01.01.2018].
S Kinoshita, S Yoshioka, and J Miyazaki. 2008. Physics of structural colors. Reports on Progress in Physics 71, 7 (jun 2008), 076401. https://doi.org/10.1088/0034-4885/71/7/ 076401

Karthik Kumar, Huigao Duan, Ravi S. Hegde, Samuel C. W. Koh, Jennifer N. Wei, and Joel K. W. Yang. 2012. Printing colour at the optical diffraction limit. Nature Nanotechnology 7, 9 (aug 2012), 557-561. https://doi.org/10.1038/nnano.2012.128

Yanxiang Lan, Yue Dong, Fabio Pellacini, and Xin Tong. 2013. Bi-scale appearance fabrication. ACM Transactions on Graphics 32, 4 (jul 2013), 1. https://doi.org/10. $1145 / 2461912.2461989$

Hye Soo Lee, Tae Soup Shim, Hyerim Hwang, Seung-Man Yang, and Shin-Hyun Kim. 2013. Colloidal Photonic Crystals toward Structural Color Palettes for Security Materials. Chemistry of Materials 25, 13 (jun 2013), 2684-2690. https://doi.org/10. 1021/cm4012603

Anat Levin, Daniel Glasner, Ying Xiong, Frédo Durand, William Freeman, Wojciech Matusik, and Todd Zickler. 2013. Fabricating BRDFs at high spatial resolution using wave optics. ACM Transactions on Graphics 32, 4 (jul 2013), 1. https://doi.org/10. $1145 / 2461912.2461981$

Jacques Louis Lions. 1971. Optimal Control of Systems Governed by Partial Differential Equations (1 ed.). Grundlehren der mathematischen Wissenschaften, Vol. 170. Springer-Verlag Berlin Heidelberg.

Wojciech Matusik, Boris Ajdin, Jinwei Gu, Jason Lawrence, Hendrik P. A. Lensch, Fabio Pellacini, and Szymon Rusinkiewicz. 2009. Printing spatially-varying reflectance. ACM Transactions on Graphics 28, 5 (dec 2009), 1. https://doi.org/10.1145/1618452. 1618474

Mariana Medina-Sánchez, Lukas Schwarz, Anne K. Meyer, Franziska Hebenstreit, and Oliver G. Schmidt. 2015. Cellular Cargo Delivery: Toward Assisted Fertilization by Sperm-Carrying Micromotors. Nano Letters 16, 1 (dec 2015), 555-561. https: //doi.org/10.1021/acs.nanolett.5b04221

Lucas R. Meza, Alex J. Zelhofer, Nigel Clarke, Arturo J. Mateos, Dennis M. Kochmann, and Julia R. Greer. 2015. Resilient 3D hierarchical architected metamaterials. Proceedings of the National Academy of Sciences 112, 37 (sep 2015), 11502-11507. https://doi.org/10.1073/pnas.1509120112

Vygantas Mizeikis. 2014. Realization of Structural Color by Direct Laser Write Technique in Photoresist. Fournal of Laser Micro/Nanoengineering 9, 1 (mar 2014), 42-45. https://doi.org/10.2961/jlmn.2014.01.0009

Michał Nawrot, Łukasz Zinkiewicz, Bartłomiej Włodarczyk, and Piotr Wasylczyk. 2013 Transmission phase gratings fabricated with direct laser writing as color filters in the visible. Optics Express 21, 26 (dec 2013), 31919. https://doi.org/10.1364/oe.21.031919

Ardavan F. Oskooi, David Roundy, Mihai Ibanescu, Peter Bermel, J.D. Joannopoulos, and Steven G. Johnson. 2010. Meep: A flexible free-software package for electromagnetic simulations by the FDTD method. Computer Physics Communications 181, 3 (mar 2010), 687-702. https://doi.org/10.1016/j.cpc.2009.11.008

Yoh-Han Pao and P. M. Rentzepis. 1965. Laser-Induced Production of Free Radicals in Organic Compounds. Applied Physics Letters 6, 5 (mar 1965), 93-95. https: //doi.org/10.1063/1.1754182

Marios Papas, Christian Regg, Wojciech Jarosz, Bernd Bickel, Philip Jackson, Wojciech Matusik, Steve Marschner, and Markus Gross. 2013. Fabricating translucent materials using continuous pigment mixtures. ACM Transactions on Graphics 32, 4 (jul 2013), 1. https://doi.org/10.1145/2461912.2461974

Chul-Soon Park, Vivek Raj Shrestha, Sang-Shin Lee, and Duk-Yong Choi. 2016. TransReflective Color Filters Based on a Phase Compensated Etalon Enabling Adjustable Color Saturation. Scientific Reports 6, 1 (may 2016), 10. https://doi.org/10.1038/ srep25496

Thiago Pereira, Carolina L. A. Paes Leme, Steve Marschner, and Szymon Rusinkiewicz. 2017. Printing anisotropic appearance with magnetic flakes. ACM Transactions on Graphics 36, 4 (jul 2017), 1-10. https://doi.org/10.1145/3072959.3073701

Andrew D. Pris, Yogen Utturkar, Cheryl Surman, William G. Morris, Alexey Vert, Sergiy Zalyubovskiy, Tao Deng, Helen T. Ghiradella, and Radislav A. Potyrailo. 2012. Towards high-speed imaging of infrared photons with bio-inspired nanoarchitectures. Nature Photonics 6, 3 (feb 2012), 195-200. https://doi.org/10.1038/nphoton.2011.355

Manuela T. Raimondi, Shane M. Eaton, Michele M. Nava, Matteo Laganà, Giulio Cerullo, and Roberto Osellame. 2012. Two-photon laser polymerization: from fundamentals to biomedical application in tissue engineering and regenerative medicine. Fournal of Applied Biomaterials \& Functional Materials 10, 1 (2012), 56-66. https://doi.org/10. 5301/jabfm.2012.9278

Louis B. Rall. 1981. Automatic Differentiation: Techniques and Applications. Lecture Notes in Computer Science, Vol. 120. Springer-Verlag Berlin Heidelberg. https: //doi.org/10.1007/3-540-10861-0

Bahaa E. A. Saleh and Malvin Carl Teich. 2001. Fundamentals of Photonics. John Wiley \& Sons, Inc. https://doi.org/10.1002/0471213748

Osamu Sato, Shoichi Kubo, and Zhong-Ze Gu. 2009. Structural Color Films with Lotus Effects, Superhydrophilicity, and Tunable Stop-Bands. Accounts of Chemical Research 42, 1 (jan 2009), 1-10. https://doi.org/10.1021/ar700197v

Tom D. Schultz and Ola M. Fincke. 2009. Structural colours create a flashing cue for sexual recognition and male quality in a Neotropical giant damselfly. Functiona Ecology 23, 4 (aug 2009), 724-732. https://doi.org/10.1111/j.1365-2435.2009.01584.x 
Bokwang Song, Villads Egede Johansen, Ole Sigmund, and Jung H. Shin. 2017. Reproducing the hierarchy of disorder for Morpho-inspired, broad-angle color reflection. Scientific Reports 7 (apr 2017), 46023. https://doi.org/10.1038/srep46023

Allen Taflove and Susan C Hagness. 2005. Computational electrodynamics: the finitedifference time-domain method (3rd ed.). Artech House.

Stéfan van der Walt, S Chris Colbert, and Gaël Varoquaux. 2011. The NumPy Array: A Structure for Efficient Numerical Computation. Computing in Science \& Engineering 13, 2 (mar 2011), 22-30. https://doi.org/10.1109/mcse.2011.37

Emma Vargo. 2017. Finding nanostructures that reproduce colors with adaptive mesh search techniques. Fournal of the Optical Society of America B 34, 10 (sep 2017), 2250. https://doi.org/10.1364/josab.34.002250

Mark Jayson Villangca, Darwin Palima, Andrew Rafael Bañas, and Jesper Glückstad. 2016. Light-driven micro-tool equipped with a syringe function. Light: Science \& Applications 5, 9 (apr 2016), e16148-e16148. https://doi.org/10.1038/lsa.2016.148

Pete Vukusic and J. Roy Sambles. 2003. Photonic structures in biology. Nature 424, 6950 (aug 2003), 852-855. https://doi.org/10.1038/nature01941

Sebastian Werner, Zdravko Velinov, Wenzel Jakob, and Matthias B. Hullin. 2017. Scratch iridescence. ACM Transactions on Graphics 36, 6 (nov 2017), 1-14. https://doi.org/ 10.1145/3130800.3130840

Tim Weyrich, Pieter Peers, Wojciech Matusik, and Szymon Rusinkiewicz. 2009. Fabricating microgeometry for custom surface reflectance. ACM Transactions on Graphics 28, 3 (jul 2009), 1. https://doi.org/10.1145/1531326.1531338

W. Wickler. 1965. Mimicry and the Evolution of Animal Communication. Nature 208, 5010 (nov 1965), 519-521. https://doi.org/10.1038/208519a0

Wilhelm Wirtinger. 1927. Zur formalen Theorie der Funktionen von mehr komplexen Veränderlichen. Math. Ann. 97, 1 (dec 1927), 357-375. https://doi.org/10.1007/ bf01447872

Richard Wollhofen, Julia Katzmann, Calin Hrelescu, Jaroslaw Jacak, and Thomas A Klar. 2013. $120 \mathrm{~nm}$ resolution and $55 \mathrm{~nm}$ structure size in STED-lithography. Optics Express 21, 9 (apr 2013), 10831. https://doi.org/10.1364/oe.21.010831

Jun Wu, Niels Aage, Sylvain Lefebvre, and Charlie Wang. 2017. Topology Optimization for Computational Fabrication. In Eurographics 2017 - Tutorials, Adrien Bousseau and Diego Gutierrez (Eds.). The Eurographics Association. https://doi.org/10.2312/ egt.20171030

Zhengmei Yang, Yanming Zhou, Yiqin Chen, Yasi Wang, Peng Dai, Zhaogang Zhang, and Huigao Duan. 2016. Reflective Color Filters and Monolithic Color Printing Based on Asymmetric Fabry-Perot Cavities Using Nickel as a Broadband Absorber Advanced Optical Materials 4, 8 (may 2016), 1196-1202. https://doi.org/10.1002/ adom.201600110

Guilian Yi and Byeng D. Youn. 2016. A comprehensive survey on topology optimization of phononic crystals. Structural and Multidisciplinary Optimization 54, 5 (jun 2016), 1315-1344. https://doi.org/10.1007/s00158-016-1520-4

Gordon Zyla, Alexander Kovalev, Evgeny L. Gurevich, Cemal Esen, Andreas Ostendorf, and Stanislav Gorb. 2018. Printing structural colors via direct laser writing. In Advanced Fabrication Technologies for Micro/Nano Optics and Photonics XI, Georg von Freymann, Winston V. Schoenfeld, and Raymond C. Rumpf (Eds.), Vol. 10544 SPIE, 9. https://doi.org/10.1117/12.2289820

\section{A SIGNAL RECONSTRUCTION}

We are free to record the time-varying complex fields $\mathbf{E}(\mathbf{x}, t)$ and/or $\mathbf{H}(\mathbf{x}, t)$ at any location $\mathbf{x}$ in the simulation domain and for the remainder of this section, we will take $E_{z}(t)$ as a representative field component. Since we use polychromatic sources, we know that $E_{z}(t)=\sum_{f \in \mathbf{f}} E_{z}(f) e^{-i \omega t}$ with $\mathbf{f}=\left(f_{1}, \ldots, f_{N_{f}}\right)$ denoting the $N_{f}$ many frequencies of the monochromatic source components. By recording $E_{z}(t)$ during a time interval $T$ after the transient phase, we get a sequence $\mathbf{s}$ of $N_{T}=\lfloor T / \Delta t\rfloor$ temporal samples, i.e.,

$$
\mathbf{s}=\left(E_{z}(0), E_{z}(\Delta t), \ldots, E_{z}\left(N_{T} \Delta t\right)\right) .
$$

For the subsequent NTFFT, we are interested in obtaining the per-frequency phasor quantities

$$
\mathbf{c}=\left(E_{z}\left(f_{1}\right), \ldots, E_{z}\left(f_{N_{f}}\right)\right)
$$

from $s$, i.e., we would like to solve the reconstruction problem

$$
\mathbf{s}=\mathbf{c V} \quad \text { with } \quad \mathbf{V}_{j k}=e^{-2 \pi i f_{j}(k-1) \Delta t}
$$

for $\mathbf{c}$. For short recording times, the Vandermonde matrix $\mathbf{V}$ is (numerically) rank-deficient and ill-conditioned, due to the tight spacing of the individual modes $\mathbf{V}_{j 2}$ on the complex unit circle [Bazán 2000].

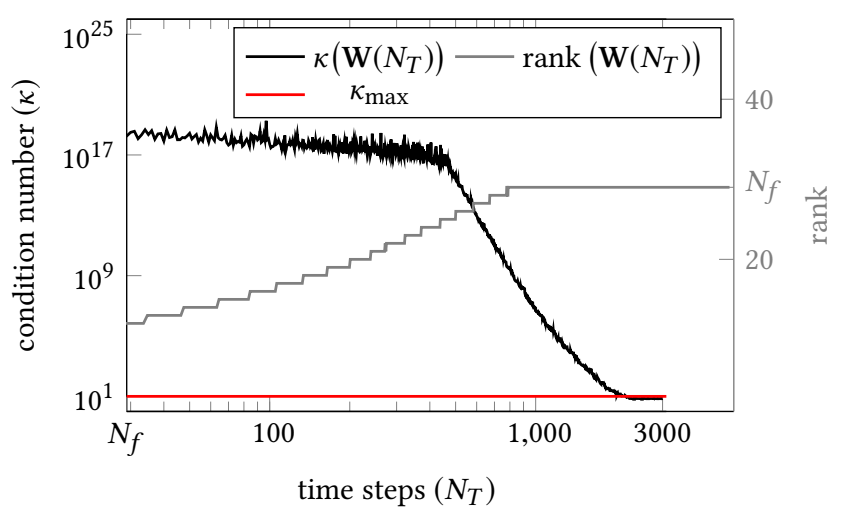

Fig. 14. Signal reconstruction. This is achieved by selecting a subset $\mathrm{W}$ of columns from $\mathrm{V}$. For $N_{f}=29$ visible light frequencies, $\approx 2200$ time steps need to be simulated such that $N_{f}$ sample times can be found that enable a full-rank reconstruction with condition number $\kappa(\mathbf{W})<\kappa_{\max }=10$. See the text for details on how to obtain $\mathrm{W}$.

This can be alleviated by recording a sufficient amount of time steps $N_{T} \gg N_{f}$; however, this leads to huge memory demands for data storage. It would be very convenient if reconstruction from only a few recorded time steps were possible. This constitutes a column subset selection problem on $\mathrm{V}$, where we search for a fullrank well-conditioned matrix $\mathbf{W}$ that consists of a minimal amount of columns of V. A solution to this problem can be found with the use of rank-revealing $\mathrm{QR}$ decomposition (RRQR) [Gu and Eisenstat 1996], by decomposing $\mathbf{V}$ into

$$
\underbrace{\mathrm{V}}_{N_{f} \times N_{T}} \overbrace{\mathbf{P}}^{N_{T} \times N_{T}}=\underbrace{\mathbf{Q}}_{N_{f} \times N_{f}} \overbrace{\mathrm{R}}^{N_{f} \times N_{T}} \text { with upper triangular } \mathrm{R} \text { and } \mathrm{Q}^{\top} \mathrm{Q}=\mathbf{1},
$$

where the permutation matrix $\mathbf{P}$ reorders the columns of $\mathbf{V}$ such that the left-most columns have the largest contribution to the orthogonal basis $\mathrm{Q}$. This makes the first $N_{f}$ columns of VP a good choice for $\mathbf{W}$ because it preserves the rank of $\mathbf{V}$ and has a similar condition number $\kappa(\mathbf{W})$ as $\mathbf{V}$ [Gu and Eisenstat 1996]. Note that this choice reduces the storage requirement from $O\left(N_{T}\right)$ to $O\left(N_{f}\right)$, i.e., two orders of magnitude in our setting.

We are left with the task of determining the required amount of time samples $N_{T}$ - a number that we want to keep as low as possible to reduce the simulation time $T=N_{T} \Delta t$ after the transient phase. By setting an upper bound $\kappa_{\max }=10$ on the permissible condition number, we obtain the desired sample count as a solution to

$$
\min N_{T} \quad \text { s.t. } \quad \operatorname{rank}(\mathbf{W})=N_{f} \text { and } \kappa(\mathbf{W}) \leq \kappa_{\max } .
$$

We solve this objective using binary search since $\kappa(\mathbf{V})$ decreases monotonously with $N_{T}$ once it has reached full rank (see Figure 14). Note that spectral culling as described in the supplemental material leads to simulations with varying numbers of frequencies $N_{f}$. We simply precompute a minimal recording time interval $T$ and the corresponding column selection $\mathbf{W}$ for each of them. 